\title{
On Kähler-like and G-Kähler-like almost Hermitian manifolds
}

https://doi.org/10.1515/coma-2020-0009

Received September 8, 2019; accepted March 13, 2020

\begin{abstract}
We introduce Kähler-like, G-Kähler-like metrics on almost Hermitian manifolds. We prove that a compact Kähler-like and G-Kähler-like almost Hermitian manifold equipped with an almost balanced metric is Kähler. We also show that if a Kähler-like and G-Kähler-like almost Hermitian manifold satisfies $B_{\bar{i} j}^{\lambda} B_{\lambda j}^{\bar{i}} \geq 0$, then the metric is almost balanced and the almost complex structure is integrable, which means that the metric is balanced. We investigate a G-Kähler-like almost Hermitian manifold under some assumptions.
\end{abstract}

Keywords: almost Hermitian manifolds, Kähler-like metrics, G-Kähler-like metrics, Chern connection

MSC 2010: 53 C15 (primary); 53C55 (secondary)

\section{Introduction}

The almost Hermitian geometry has been studied vigorously in last years such as in [14], [15], [16], [24] and [26]. We introduce the notion of a Kähler-like almost Hermitian metric, a G-Kähler-like almost Hermitian metric and an almost balanced metric and investigate compact almost Hermitian manifolds equipped with these three conditions. In the Hermitian case, Yang and Zheng examined the Hermitian curvature tensors of Hermitian metrics, as the curvature tensors satisfies all the symmetry conditions of the curvature tensor of a Kähler metric in [23]. They called these metrics Kähler-like. When a manifold is compact, these metrics are more special than balanced metrics since they are always balanced, that is, $d\left(\omega^{n-1}\right)=0$, where $\omega$ is the fundamental 2-form associated to a Hermitian metric and $n$ is the complex dimension of the manifold. This fact has attracted attention in the reserch of non-Kähler Calabi-Yau manifolds. Their definitions are as follows. Given a Hermitian manifold $\left(M^{n}, J, g\right)$, there are two canonical connections associated to $g$, the Chern connection $\nabla$ and the Levi-Civita connection $D$. Denote $R$ and $R^{L}$ the curvature tensor of these two connections respectively. Notice that in this whole paper, in the almost Hermitian case $M^{2 n}$ indicates that $2 n=\operatorname{dim}_{\mathbb{R}} M$, in the Hermitian case $M^{n}$ means that $n=\operatorname{dim}_{\mathbb{C}} M$.

Definition 1.1. (Kähler-like and G-Kähler-like [23]) A Hermitian metric $g$ will be called Kähler-like, if $R_{X \bar{Y} Z \bar{W}}=$ $R_{Z \bar{Y} X \bar{W}}$ holds for any type $(1,0)$ tangent vectors $X, Y, Z$ and $W$. Similarly, if $R_{X Y \bar{Z} \bar{W}}^{L}=R_{X Y Z \bar{W}}^{L}=0$ for any type $(1,0)$ tangent vectors $X, Y, Z$ and $W$, we will say that $g$ is Gray-Kähler-like, or $G$-Kähler-like for short.

The G-Kähler-like condition was firstly introduced by Gray in [11]. Yang and Zheng showed that when $R=$ $R^{L}$, then $g$ is Kähler in [23, Theorem 1.1], and they also showed that when the manifold is compact, either condition, the Kähler-likeness or the G-Kähler-likeness, would imply that the metric is balanced.

Proposition 1.1. ([23, Theorem 1.3]) Let $\left(M^{n}, J, g\right)$ be a compact Hermitian manifold. If it is either Kähler-like or G-Kähler-like, then it must be balanced.

${ }^{\star}$ Corresponding Author: Masaya Kawamura: Department of Social Design Engineering, National Institute of Technology, Kochi College,200-1, Otsu, Monobe, Nankoku, Kochi, Japan 783-8508 E-mail: kawamura@kochi-ct.ac.jp 
In this sense, the Kähler-likeness and G-Kähler-likeness are more special classes of Hermitian metrics than being balanced for compact Hermitian manifolds. Notice that in the case of $n=2$, a compact Kähler-like or G-Kähler-like Hermitian surface $\left(M^{2}, J, g\right)$ is Kähler. Note that Vaisman has already showed that any compact G-Kähler-like Hermitian surface is Kähler in [21].

We now extend the analysis to almost Hermitian geometry. Let $(M, J)$ be an almost complex manifold and let $g$ be an almost Hermitian metric on $M$. Let $\left\{Z_{r}\right\}$ be an arbitrary local $(1,0)$-frame around a fixed point $p \in M$ and let $\left\{\zeta^{r}\right\}$ be the associated coframe. Then the associated real $(1,1)$-form $\omega$ with respect to $g$ takes the local expression $\omega=\sqrt{-1} g_{r \bar{k}} \zeta^{r} \wedge \zeta^{\bar{k}}$. We will also refer to $\omega$ as to an almost Hermitian metric.

We define a Kähler-like almost Hermitian metric in the following as in [23, Definition (Kähler-like and G-Kähler-like)]. $R^{L}$ denotse the curvature tensor with respect to the Levi-Civita connection $D$ on an almost Hermitian manifold. Here, $R^{L}$ is just the Riemannian curvature tensor, and we extend it linearly over $\mathbb{C} . R^{L}$ is anti-symmetric with respect to their first two or last two positions and symmetric when its first two and last two positions are interchanged, and satisfies the Bianchi identity which means that when one positions is held fixed while the other three are cyclicly permuted, the sum is always zero.

We denote by $\Omega$ the curvature of the Chern connection $\nabla$ on an almost Hermitian manifold. We can regard $\Omega$ as a section of $\Lambda^{2} M \otimes T M, \Omega \in \Gamma\left(\Lambda^{2} M \otimes T M\right)$ and $\Omega$ splits in

$$
\Omega=\Omega^{(2,0)}+\Omega^{(1,1)}+\Omega^{(0,2)}=H+R+\bar{H} .
$$

Definition 1.2. Given an almost Hermitian manifold $\left(M^{2 n}, J, g\right)$, almost Hermitian metric $g$ will be called Kähler-like, if $R_{X \bar{Y} Z \bar{W}}=R_{Z \bar{Y} X \bar{W}}$ for any $(1,0)$-tangent vectors $X, Y, Z$ and $W$. When the almost Hermitian metric $g$ is Kähler-like, the triple $\left(M^{2 n}, J, g\right)$ will be called a Kähler-like almost Hermitian manifold. Similarly, if $R_{X Y \bar{Z} \bar{W}}^{L}=R_{X Y Z \bar{W}}^{L}=0$ for any type $(1,0)$ tangent vectors $X, Y, Z$ and $W$, we will say that $g$ is G-Kähler-like. When the almost Hermitian metric $g$ is G-Kähler-like, the triple $\left(M^{2 n}, J, g\right)$ will be called a G-Kähler-like almost Hermitian manifold.

When $g$ is Kähler-like, by taking complex conjugations, we see that $R$ is also symmetric with respect to its second and fourth positions, thus obeying all the symmetries of the curvature tensor of a Kähler metric. There are non-Kähler almost Hermitian metrics $g$ which are Kähler-like. For instance, there are almost Hermitian manifolds that are non-Kähler but with $R=0$ everywhere. Such manifolds are obviously Kähler-like. The Iwasawa manifold is the unique example of a 6-dimensional non-Kähler almost complex nilmanifold admitting a quasi-Kähler $R$-flat metric (cf. [6], [23]). One can find some almost Hermitian Chern-flat examples such that $J$ is not integrable in [6, Corollary 1.3].

We next define an almost balanced metric.

Definition 1.3. Let $\left(M^{2 n}, J\right)$ be an almost complex manifold. A metric $g$ is called an almost balanced metric on $M$ if $g$ is an almost Hermitian metric whose associated real $(1,1)$-form $\omega=\sqrt{-1} g_{i j} \zeta^{i} \wedge \zeta^{j}$ satisfies $d\left(\omega^{n-1}\right)=0$. When an almost Hermitian metric $g$ is almost balanced, the triple $\left(M^{2 n}, J, g\right)$ will be called an almost balanced manifold.

We have shown the following generalized result of Proposition 1.1 under an additional assumption for the almost complex structure $J$.

Proposition 1.2. ([15, Theorem 1.1]) Let $\left(M^{2 n}, J, g\right)$ be a compact Kähler-like almost Hermitian manifold with $n \geq 2$. Then $\left(M^{2 n}, J, g\right)$ is almost balanced if and only if $B_{i k}^{\bar{q}} B_{\bar{q} \bar{l}}^{i}=0$ for all $k, l=1, \ldots, n$.

Note that $B_{i k}^{\bar{q}}$,s do not depend on $g$, depend only on $J$, where $B_{i k}^{\bar{q}}$, s are the structure coefficients of Lie bracket such that $B_{i k}^{\bar{q}}=-N_{i k}^{\bar{q}}=-T_{i k}^{\bar{q}}, N_{i k}^{\bar{q}}$, s are the coefficients of the Nijenhuis tensor of $J$ and $T_{i k}^{\bar{q}}$,s are the coefficients of torsion of the Chern connection. See p.9 for a detailed discussion.

A quasi-Kähler structure is an almost Hermitian structure whose real $(1,1)$-form $\omega$ satisfies $(d \omega)^{(1,2)}=$ $\bar{\partial} \omega=0$, which is equivalent to the original definition of quasi-Kählerity: $D_{X} J(Y)+D_{J X} J(J Y)=0$ for all vector fields $X, Y$ (cf. [10]). An almost Kähler structure is an almost Hermitian structure whose real $(1,1)$-form $\omega$ 
satisfies $d \omega=0$. It is important for us to study quasi-Kähler manifolds since they include the classes of almost Kähler manifolds and nearly Kähler manifolds. Recall that an almost Hermitian manifold $(M, J, g)$ is said to be nearly Kähler if $\left(D_{X} J\right) X=0$ for any tangent vector field $X$ and $D J \neq 0$, where $D$ is the Levi-Civita connection associated to $g$. An almost Kähler or quasi-Kähler manifold with $J$ integrable is a Kähler manifold. We have shown the following result on compact Kähler-like almost Hermitian manifolds.

Proposition 1.3. ([16, Theorem 1.1]) Let $\left(M^{2 n}, J, g\right)$ be a compact Kähler-like almost Hermitian manifold with $n \geq 2$. If $M^{2 n}$ admits a positive $\partial \bar{\partial}$-closed $(n-2, n-2)$-form $\chi$, then $g$ is quasi-Kähler. In particular, if $M^{2 n}$ is compact, Kähler-like, and $\partial \bar{\partial}\left(\omega^{n-2}\right)=0$, then $g$ is quasi-Kähler. When $n=2$, compactness implies that any Kähler-like metric is almost Kähler.

Note that in dimension 4, every quasi-Kähler manifold is almost Kähler. In general, there are known examples of quasi-Kähler manifolds which are not almost Kähler. The canonical example of a quasi-Kähler Chern-flat manifold is the Iwasawa manifold $M$ equipped with the almost Hermitian structure associated to the almost complex structure $J_{3}$ defined in [1]. The structure $J_{3}$ is the unique invariant almost complex structure on $M$ such that its associated fundamental form $\omega_{3}$ with respect to a canonical metric of $M$ is quasi-Kähler and not-symplectic (cf. [7]).

Yang and Zheng raise the natural question of whether a Kähler-like and G-Kähler-like Hermitian manifold is Kähler or not. They gave the following partial answer to this question.

Proposition 1.4. ([23, Theorem 1.2]) Let $\left(M^{n}, J, g\right)$ be a Hermitian manifold that is both Kähler-like and GKähler-like. If either $M^{n}$ is compact or $n \leq 3$, then $g$ is Kähler.

We prove the generalized result of Proposition 1.4.

Theorem 1.1. Let $\left(M^{2 n}, J, g\right)$ be a compact Kähler-like and G-Kähler-like almost balanced manifold with $n \geq$ 2. Then $g$ is Kähler.

By applying Proposition 1.3 and Theorem 1.1, we get the following corollary.

Corollary 1.1. A 4-dimensional compact Kähler-like and G-Kähler-like almost Hermitian manifold is Kähler.

We show the following result.

Theorem 1.2. Let $\left(M^{2 n}, J, g\right)$ be a G-Kähler-like almost Hermitian manifold with $n \geq 2$. Assume that $\nabla_{\bar{i}} w_{i}+$ $B_{i j}^{\lambda} B_{\lambda j}^{\bar{i}} \geq 0$. Then the metric $g$ is almost balanced and the almost complex structure $J$ is integrable (i.e. $g$ is balanced).

From Lemma 4.2, we have $\nabla_{\bar{j}} w_{j}=B_{\bar{i}} B_{\lambda j}^{\bar{i}}$ on a Kähler-like almost Hermitian manifold. By combining this formula and Theorem 1.2, we obtain the following result.

Corollary 1.2. Let $\left(M^{2 n}, J, g\right)$ be a Kähler-like and G-Kähler-like almost Hermitian manifold with $n \geq 2$. Assume that $B_{i j}^{\lambda} B_{\lambda j}^{\bar{i}} \geq 0$. Then the metric $g$ is almost balanced and the almost complex structure $J$ is integrable (i.e. $g$ is balanced).

This paper is organized as follows: in the second section, we recall some basic definitions and computations. In the third section, we define Kähler-likeness and G-Kähler-likeness in another way. And we show some equivalences between the definitions in Definition 1.2 and the ones in Definition 3.1. In the fourth section, by calculating the curvature tensor applying Proposition 1.2 and 1.4, we show Theorem 1.1 and 1.2. In the last section, we study the case when $R_{i \bar{j} k \bar{l}}=R_{i \bar{j} k \bar{l}}^{L}$ for all $i, j, k, l=1, \ldots, n$ and $B_{\bar{i} \bar{j}}^{\lambda} B_{\lambda j}^{\bar{i}}=0$, and then we give a proof of Theorem 3.1. Notice that we assume the Einstein convention omitting the symbol of sum over repeated indexes in all this paper. 


\section{Preliminaries}

\subsection{The Nijenhuis tensor of the almost complex structure}

Let $M$ be a $2 n$-dimensional smooth differentiable manifold. An almost complex structure on $M$ is an endomorphism $J$ of $T M, J \in \Gamma(\operatorname{End}(T M))$, satisfying $J^{2}=-I d_{T M}$. The pair $(M, J)$ is called an almost complex manifold. Let $(M, J)$ be an almost complex manifold. We define a bilinear map on $C^{\infty}(M)$ for $X, Y \in \Gamma(T M)$ by

$$
4 N(X, Y):=[J X, J Y]-J[J X, Y]-J[X, J Y]-[X, Y],
$$

which is the Nijenhuis tensor of $J$. The Nijenhuis tensor $N$ satisfies $N(X, Y)=-N(Y, X), N(J X, Y)=-J N(X, Y)$, $N(X, J Y)=-J N(X, Y), N(J X, J Y)=-N(X, Y)$. For any $(1,0)$-vector fields $W$ and $V, N(V, W)=-[V, W]^{(0,1)}$, $N(V, \bar{W})=N(\bar{V}, W)=0$ and $N(\bar{V}, \bar{W})=-[\bar{V}, \bar{W}]^{(1,0)}$ since we have $4 N(V, W)=-2([V, W]+\sqrt{-1} J[V, W])$, $4 N(\bar{V}, \bar{W})=-2([\bar{V}, \bar{W}]-\sqrt{-1} J[\bar{V}, \bar{W}])$. An almost complex structure $J$ is called integrable if $N=0$ everywhere on $M$. Giving a complex structure on a differentiable manifold $M$ is equivalent to giving an integrable almost complex structure on $M$ (cf. [18]). Let $(M, J)$ be an almost complex manifold. A Riemannian metric $g$ on $M$ is called $J$-invariant if $J$ is compatible with $g$, i.e., for any $X, Y \in \Gamma(T M), g(X, Y)=g(J X, J Y)$. In this case, the pair $(J, g)$ is called an almost Hermitian structure. The fundamental 2-form $\omega$ associated to a $J$-invariant Riemannian metric $g$, i.e., an almost Hermitian metric, is determined by, for $X, Y \in \Gamma(T M), \omega(X, Y)=g(J X, Y)$. Indeed we have, for any $X, Y \in \Gamma(T M)$,

$$
\omega(Y, X)=g(J Y, X)=g\left(J^{2} Y, J X\right)=-g(J X, Y)=-\omega(X, Y)
$$

and $\omega \in \Gamma\left(\bigwedge^{2} T^{\star} M\right)$. We will also refer to the associated real fundamental $(1,1)$-form $\omega$ as an almost Hermitian metric. The form $\omega$ is related to the volume form $d V_{g}$ by $n ! d V_{g}=\omega^{n}$. Let a local $(1,0)$-frame $\left\{Z_{r}\right\}$ on $(M, J)$ with an almost Hermitian metric $g$ and let $\left\{\zeta^{r}\right\}$ be a local associated coframe with respect to $\left\{Z_{r}\right\}$, i.e., $\zeta^{i}\left(Z_{j}\right)=\delta_{j}^{i}$ for $i, j=1, \ldots, n$. Since $g$ is almost Hermitian, its components satsfy $g_{i j}=g_{\overline{i j}}=0$ and $g_{i \bar{j}}=g_{\overline{j i}}=\bar{g}_{\overline{i j}}$.

With using these local frame $\left\{Z_{r}\right\}$ and coframe $\left\{\zeta^{r}\right\}$, we have

$$
N\left(Z_{\bar{i}}, Z_{\bar{j}}\right)=-\left[Z_{\bar{i}}, Z_{\bar{j}}\right]^{(1,0)}=: N N_{\overline{i j}}^{k} Z_{k}, \quad N\left(Z_{i}, Z_{j}\right)=-\left[Z_{i}, Z_{j}\right]^{(0,1)}=\overline{N_{\bar{i} j}^{k}} Z_{\bar{k}},
$$

and

$$
N=\frac{1}{2} \overline{N_{i j}^{k}} Z_{\bar{k}} \otimes\left(\zeta^{i} \wedge \zeta^{j}\right)+\frac{1}{2} N_{\overline{i j}}^{k} Z_{k} \otimes\left(\zeta^{\bar{i}} \wedge \zeta^{\bar{j}}\right) .
$$

We write $T^{\mathbb{R}} M$ for the real tangent space of $M$. Then its complexified tangent space is given by $T^{\mathbb{C}} M=$ $T^{\mathbb{R}} M \otimes_{\mathbb{R}} \mathbb{C}$. By extending $J \mathbb{C}$-linearly and $g, \omega$, $\mathbb{C}$-bilinearly to $T^{\mathbb{C}} M$, they are also defined on $T^{\mathbb{C}} M$ and we observe that the complexified tangent space $T^{\mathbb{C}} M$ can be decomposed as $T^{\mathbb{C}} M=T^{1,0} M \oplus T^{0,1} M$, where $T^{1,0} M, T^{0,1} M$ are the eigenspaces of $J$ corresponding to eigenvalues $\sqrt{-1}$ and $-\sqrt{-1}$, respectively:

$$
T^{1,0} M=\{X-\sqrt{-1} J X \mid X \in T M\}, \quad T^{0,1} M=\{X+\sqrt{-1} J X \mid X \in T M\} .
$$

Let $\Lambda^{r} M=\bigoplus_{p+q=r} \Lambda^{p, q} M$ for $0 \leq r \leq 2 n$ denote the decomposition of complex differential $r$-forms into $(p, q)$-forms, where $\Lambda^{p, q} M=\Lambda^{p}\left(\Lambda^{1,0} M\right) \otimes \Lambda^{q}\left(\Lambda^{0,1} M\right)$,

$$
\Lambda^{1,0} M=\left\{\alpha+\sqrt{-1} J \alpha \mid \alpha \in \Lambda^{1} M\right\}, \quad \Lambda^{0,1} M=\left\{\alpha-\sqrt{-1} J \alpha \mid \alpha \in \Lambda^{1} M\right\}
$$

and $\Lambda^{1} M$ denotes the dual of $T M$.

Let $\left(M^{2 n}, J, g\right)$ be an almost Hermitian manifold. An affine connection $D$ on $T M$ is called almost Hermitian connection if $D g=D J=0$. For the almost Hermitian connection, we have the following Lemma (cf. [4], [9], [22], [26]).

Lemma 2.1. Let $(M, J, g)$ be an almost Hermitian manifold with $\operatorname{dim}_{\mathbb{R}} M=2 n$. Then for any given vector valued $(1,1)$-form $\Theta=\left(\Theta^{i}\right)_{1 \leq i \leq n}$, there exists a unique almost Hermitian connection $D$ on $(M, J, g)$ such that the $(1,1)$-part of the torsion is equal to the given $\Theta$. 
If the $(1,1)$-part of the torsion of an almost Hermitian connection vanishes everywhere, then the connction is called the second canonical connection or the Chern connection. We will refer the connection as the Chern connection and denote it by $\nabla$.

Now let $\nabla$ be the Chern connection on $M$. We denote the structure coefficients of Lie bracket by

$$
\begin{gathered}
{\left[Z_{i}, Z_{j}\right]=: B_{i j}^{r} Z_{r}+B_{i j}^{\bar{r}} Z_{\bar{r}}=B_{i j}^{r} Z_{r}-\overline{N_{i \bar{j}}^{r}} Z_{\bar{r}}, \quad\left[Z_{i}, Z_{\bar{j}}\right]=: B_{i \bar{j}}^{r} Z_{r}+B_{i \bar{j}}^{\bar{r}} Z_{\bar{r}},} \\
{\left[Z_{\bar{i}}, Z_{\bar{j}}\right]=: B_{i \bar{j}}^{r} Z_{r}+B_{i \bar{j}}^{\bar{r}} Z_{\bar{r}}=-N_{i \bar{i}}^{r} Z_{r}+B_{\bar{i} j}^{\bar{r}} Z_{\bar{r}},}
\end{gathered}
$$

where we used that $\left[Z_{i}, Z_{j}\right]^{(0,1)}=-\overline{N_{i j}^{r}} Z_{\bar{r}},\left[Z_{\bar{i}}, Z_{\bar{j}}\right]^{(1,0)}=-N_{i j}^{r} Z_{r}$ and then $B_{i j}^{\bar{r}}=-\overline{N_{i j}^{r}}, B_{i j}^{r}=-N_{i \bar{j}}^{r}$. Also we here note that for instance, $\left[Z_{i}, Z_{\bar{j}}\right]=\left[Z_{i}, Z_{\bar{j}}\right]^{(1,0)}+\left[Z_{i}, Z_{\bar{j}}\right]^{(0,1)}$, where

$$
\left[Z_{i}, Z_{\bar{j}}\right]^{(1,0)}=\frac{1}{2}\left(\left[Z_{i}, Z_{\bar{j}}\right]-\sqrt{-1} J\left[Z_{i}, Z_{\bar{j}}\right]\right), \quad\left[Z_{i}, Z_{\bar{j}}\right]^{(0,1)}=\frac{1}{2}\left(\left[Z_{i}, Z_{\bar{j}}\right]+\sqrt{-1} J\left[Z_{i}, Z_{\bar{j}}\right]\right) .
$$

Notice that $J$ is integrable if and only if the $B_{i j}^{\bar{r}}$ 's vanish.

Note that for any $p$-form $\psi$, there holds that

$$
\begin{aligned}
d \psi\left(X_{1}, \ldots, X_{p+1}\right)= & \sum_{i=1}^{p+1}(-1)^{i+1} X_{i}\left(\psi\left(X_{1}, \ldots, \widehat{X}_{i}, \ldots, X_{p+1}\right)\right) \\
& +\sum_{i<j}(-1)^{i+j} \psi\left(\left[X_{i}, X_{j}\right], X_{1}, \ldots, \widehat{X}_{i}, \ldots, \widehat{X}_{j}, \ldots, X_{p+1}\right)
\end{aligned}
$$

for any vector fields $X_{1}, \ldots, X_{p+1}$ on $M$ (cf. [26]). We directly compute that

$$
d \zeta^{s}=-\frac{1}{2} B_{k l}^{s} \zeta^{k} \wedge \zeta^{l}-B_{k l}^{s} \zeta^{k} \wedge \zeta^{\bar{l}}+\frac{1}{2} N_{\bar{k} l}^{s} \zeta^{\bar{k}} \wedge \zeta^{\bar{l}}
$$

According to the direct computation above, we may split the exterior differential operator $d: \Lambda^{p} M \otimes_{\mathbb{R}} \mathbb{C} \rightarrow$ $\Lambda^{p+1} M \otimes_{\mathbb{R}} \mathbb{C}$, into four components

$$
d=A+\partial+\bar{\partial}+\bar{A}
$$

with

$$
\begin{gathered}
\partial: \Lambda^{p, q} M \rightarrow \Lambda^{p+1, q} M, \quad \bar{\partial}: \Lambda^{p, q} M \rightarrow \Lambda^{p, q+1} M, \\
A: \Lambda^{p, q} M \rightarrow \Lambda^{p+2, q-1} M, \quad \bar{A}: \Lambda^{p, q} M \rightarrow \Lambda^{p-1, q+2} M,
\end{gathered}
$$

since we have

$$
d\left(\Gamma\left(\Lambda^{r, s} M\right)\right) \subseteq \Gamma\left(\Lambda^{r+2, s-1} M \oplus \Lambda^{r+1, s} M \oplus \Lambda^{r, s+1} M \oplus \Lambda^{r-1, s+2} M\right) .
$$

In terms of these components, the condition $d^{2}=0$ can be written as

$$
\begin{gathered}
A^{2}=0, \quad \partial A+A \partial=0, \bar{\partial} \bar{A}+\bar{A} \bar{\partial}=0, \quad \bar{A}^{2}=0, \\
A \bar{\partial}+\partial^{2}+\bar{\partial} A=0, \quad A \bar{A}+\partial \bar{\partial}+\bar{\partial} \partial+\bar{A} A=0, \quad \partial \bar{A}+\bar{\partial}^{2}+\bar{A} \partial=0 .
\end{gathered}
$$

Notice that $J$ is integrable if and only if $A=0$, equivalently, if and only if $\bar{\partial}^{2}=0$.

For any real $(1,1)$-form $\rho=\sqrt{-1} \rho_{i \bar{j}} \zeta^{i} \wedge \zeta^{\bar{j}}$, we have

$$
\begin{aligned}
& \bar{\partial} \rho=\frac{\sqrt{-1}}{2}\left(Z_{\bar{j}}\left(\rho_{k \bar{i}}\right)-Z_{\bar{i}}\left(\rho_{k \bar{j}}\right)-B_{k \bar{i}}^{s} \rho_{s \bar{j}}+B_{k \bar{j}}^{s} \rho_{s \bar{i}}+B_{\bar{i} j}^{\bar{s}} \rho_{k \bar{s}}\right) \zeta^{k} \wedge \zeta^{\bar{i}} \wedge \zeta^{\bar{j}}, \\
& \partial \rho=\frac{\sqrt{-1}}{2}\left(Z_{i}\left(\rho_{j \bar{k}}\right)-Z_{j}\left(\rho_{i \bar{k}}\right)-B_{i j}^{s} \rho_{s \bar{k}}-B_{i \bar{k}}^{\bar{s}} \rho_{j \bar{s}}+B_{j \bar{k}}^{\bar{s}} \rho_{i \bar{s}}\right) \zeta^{i} \wedge \zeta^{j} \wedge \zeta^{\bar{k}} .
\end{aligned}
$$

From these computations above, we have

$$
\bar{\partial} \omega=\frac{\sqrt{-1}}{2}\left(Z_{\bar{j}}\left(g_{k \bar{i}}\right)-Z_{\bar{i}}\left(g_{k \bar{j}}\right)-B_{k \bar{i}}^{s} g_{s \bar{j}}+B_{k \bar{j}}^{s} g_{s \bar{i}}+B_{\bar{i} \bar{j}}^{\bar{s}} g_{k \bar{s}}\right) \zeta^{k} \wedge \zeta^{\bar{i}} \wedge \zeta^{\bar{j}}=\frac{\sqrt{-1}}{2} T_{\overline{j i} k} \zeta^{k} \wedge \zeta^{\bar{i}} \wedge \zeta^{\bar{j}}
$$

and

$$
\partial \omega=\frac{\sqrt{-1}}{2}\left(Z_{i}\left(g_{j \bar{k}}\right)-Z_{j}\left(g_{i \bar{k}}\right)-B_{i j}^{S} g_{s \bar{k}}-B_{i \bar{k}}^{\bar{S}} g_{j \bar{s}}+B_{j \bar{k}}^{\bar{S}} g_{i \bar{s}}\right) \zeta^{i} \wedge \zeta^{j} \wedge \zeta^{\bar{k}}=\frac{\sqrt{-1}}{2} T_{i j \bar{k}} \zeta^{i} \wedge \zeta^{j} \wedge \zeta^{\bar{k}}
$$




\subsection{The torsion and the curvature on almost complex manifolds}

Let a local $(1,0)$-frame $\left\{Z_{r}\right\}$ on $(M, J)$ with an almost Hermitian metric $g$ and let $\left\{\zeta^{r}\right\}$ be a local associated coframe with respect to $\left\{Z_{r}\right\}$. Since the Chern connection $\nabla$ preserves $J$, we are able to define the Christoffel symbols: for $i, j, r=1, \ldots, n$,

$$
\nabla_{i} Z_{j}=\nabla_{Z_{i}} Z_{j}=\Gamma_{i j}^{r} Z_{r}, \quad \nabla_{i} Z_{\bar{j}}=\nabla_{Z_{i}} Z_{\bar{j}}=\Gamma_{i \bar{j}}^{\bar{r}} Z_{\bar{r}},
$$

where

$$
\Gamma_{i j}^{r}=g^{r \bar{s}} Z_{i}\left(g_{j \bar{s}}\right)-g^{r \bar{s}} g_{j \bar{l}} B_{i \bar{s}}^{\bar{l}}, \quad \Gamma_{i p}^{p}=Z_{i}(\log \operatorname{det} g)-B_{i \bar{s}}^{\bar{s}} .
$$

The torsion $T=\left(T^{i}\right)$ of the Chern connection $\nabla$ is defined by

$$
T^{i}:=d \zeta^{i}-\zeta^{p} \wedge \gamma_{p}^{i}, \quad T^{\bar{i}}:=d \zeta^{\bar{i}}-\zeta^{\bar{p}} \wedge \gamma_{\bar{p}}^{\bar{i}},
$$

where $\gamma=\left(\gamma_{j}^{i}\right)$ is the connection form defined by $\gamma_{j}^{i}:=\Gamma_{k j}^{i} \zeta^{k}+\Gamma_{\bar{k} j}^{i} \zeta^{\bar{k}}$. Since the torsion $T$ of the Chern connection $\nabla$ has no (1, 1)-part;

$$
\begin{aligned}
& 0=T_{k \bar{l}}^{i}=T^{i}\left(Z_{k}, Z_{\bar{l}}\right)=-\zeta^{i}\left(\left[Z_{k}, Z_{\bar{l}}\right]\right)-\left(\Gamma_{s p}^{i} \zeta^{p} \wedge \zeta^{s}+\Gamma_{\bar{s} p}^{i} \zeta^{p} \wedge \zeta^{\bar{s}}\right)\left(Z_{k}, Z_{\bar{l}}\right)=-B_{k \bar{l}}^{i}-\Gamma_{\bar{l} k}^{i} \\
& 0=T_{k \bar{l}}^{\bar{i}}=T^{\bar{i}}\left(Z_{k}, Z_{\bar{l}}\right)=-\zeta^{\bar{i}}\left(\left[Z_{k}, Z_{\bar{l}}\right]\right)-\left(\Gamma_{s \bar{p}}^{\bar{i}} \zeta^{\bar{p}} \wedge \zeta^{s}+\Gamma_{\bar{s} \bar{p}}^{\bar{i}} \zeta^{\bar{p}} \wedge \zeta^{\bar{s}}\right)\left(Z_{k}, Z_{\bar{l}}\right)=-B_{k \bar{l}}^{\bar{i}}+\Gamma_{k \bar{l}}^{\bar{i}},
\end{aligned}
$$

we have

$$
\Gamma_{i \bar{j}}^{\bar{r}}=B_{i \bar{j}}^{\bar{r}} \text {. }
$$

Here note that $B_{j \bar{b}}^{\bar{q}}, B_{\bar{j} b}^{q}$,s do not depend on $g$, which depend only on $J$ since the mixed derivatives $\nabla_{j} Z_{\bar{b}}$ do not depend on $g$ (cf. [22]).

The torsion $T$ of $\nabla$ has no $(1,1)$-part and the only non-vanishing components are as follows:

$$
\begin{gathered}
T_{i j}^{S}=T^{s}\left(Z_{i}, Z_{j}\right)=-\zeta^{S}\left(\left[Z_{i}, Z_{j}\right]\right)-\left(\Gamma_{s p}^{i} \zeta^{p} \wedge \zeta^{s}+\Gamma_{\bar{s} p}^{i} \zeta^{p} \wedge \zeta^{\bar{s}}\right)\left(Z_{i}, Z_{j}\right)=-B_{i j}^{S}-\Gamma_{j i}^{S}+\Gamma_{i j}^{S}, \\
T_{i j}^{\bar{s}}=d \zeta^{\bar{s}}\left(Z_{i}, Z_{j}\right)=-\zeta^{\bar{s}}\left(\left[Z_{i}, Z_{j}\right]\right)=-B_{i j}^{\bar{s}}
\end{gathered}
$$

and on the other hand we have $d \zeta^{\bar{s}}\left(Z_{i}, Z_{j}\right)=N_{i j}^{\bar{s}}$, hence we obtain that $T_{i j}^{\bar{s}}=N_{i j}^{\bar{s}}=-B_{i j}^{\bar{s}}$. These computations tell us that $T$ splits in

$$
T=T^{\prime}+T^{\prime \prime},
$$

where $T^{\prime} \in \Gamma\left(\Lambda^{2,0} M \otimes T^{1,0} M\right)$, a section of $\Lambda^{2,0} M \otimes T^{1,0} M$, and $T^{\prime \prime} \in \Gamma\left(\Lambda^{2,0} M \otimes T^{0,1} M\right)$. The torsion $T=\left(T^{i}\right)$ can be split into $T=T^{2,0}+T^{1,1}+T^{0,2}=T^{2,0}+T^{0,2}$ since $T^{1,1}=0$, where $T^{2,0}=\left(\frac{1}{2} T_{j k}^{i} \zeta^{j} \wedge \zeta^{k}\right)_{1 \leq i \leq n}$, $T^{0,2}=\left(\frac{1}{2} N_{\bar{j} \bar{k}}^{i} \zeta^{\bar{j}} \wedge \zeta^{\bar{k}}\right)_{1 \leq i \leq n}$, which tells us that (0,2)-part of the Chern connection is uniquely determined by the Nijenhuis tensor (cf. [26]). Let $(M, J, g)$ be an almost Hermitian manifold. Let $\left\{Z_{r}\right\}$ be a local $(1,0)$-frame with respect to $g$ and let $\left\{\zeta^{r}\right\}$ be the associated coframe. Then the associated real $(1,1)$-form $\omega$ with respect to $g$ takes the local expression $\omega=\sqrt{-1} \zeta^{r} \wedge \zeta^{\bar{r}}$. With using these notations, we may compute

$$
\begin{aligned}
d \omega= & \sqrt{-1}\left(d \zeta^{r} \wedge \zeta^{\bar{r}}-\zeta^{r} \wedge d \zeta^{\bar{r}}\right) \\
= & \sqrt{-1}\left\{\left(-\gamma_{p}^{r} \wedge \zeta^{p}+T^{r}\right) \wedge \zeta^{\bar{r}}-\zeta^{r} \wedge\left(-\gamma_{\bar{p}}^{\bar{r}} \wedge \zeta^{\bar{p}}+T^{\bar{r}}\right)\right\} \\
= & \sqrt{-1}\left(-\gamma_{p}^{r} \wedge \zeta^{p} \wedge \zeta^{\bar{r}}+T^{r} \wedge \zeta^{\bar{r}}-\zeta^{r} \wedge \gamma_{r}^{p} \wedge \zeta^{\bar{p}}-\zeta^{r} \wedge T^{\bar{r}}\right) \\
= & \sqrt{-1}\left(T^{r} \wedge \zeta^{\bar{r}}-\zeta^{r} \wedge T^{\bar{r}}-\gamma_{p}^{r} \wedge \zeta^{p} \wedge \zeta^{\bar{r}}+\gamma_{r}^{p} \wedge \zeta^{r} \wedge \zeta^{\bar{p}}\right) \\
= & \sqrt{-1}\left(T^{r} \wedge \zeta^{\bar{r}}-\zeta^{r} \wedge T^{\bar{r}}\right) \\
= & \frac{\sqrt{-1}}{2}\left(N_{\bar{i} j}^{k} \zeta^{\bar{k}} \wedge \zeta^{\bar{i}} \wedge \zeta^{\bar{j}}-\overline{N_{i j}^{k}} \zeta^{k} \wedge \zeta^{i} \wedge \zeta^{j}\right. \\
& \left.+T_{i j}^{k} \zeta^{\bar{k}} \wedge \zeta^{i} \wedge \zeta^{j}+T_{j i}^{\bar{k}} \zeta^{k} \wedge \zeta^{\bar{i}} \wedge \zeta^{\bar{j}}\right),
\end{aligned}
$$


where we used the skew-Hermitian property $\gamma_{r}^{p}+\gamma_{\bar{p}}^{\bar{r}}=0$, which can be obtained with using $\nabla g=0$ (cf. [21]). Note that we obtain for any $(1,0)$-form $\beta$,

$$
(\bar{\partial} \beta)_{k \bar{j}}=Z_{\bar{j}}\left(\beta_{k}\right)-B_{\bar{j} k}^{m} \beta_{m}=\nabla_{\bar{j}} \beta_{k}, \quad \bar{\partial} \beta=\nabla_{\bar{j}} \beta_{k} \zeta^{\bar{j}} \wedge \zeta^{k}
$$

and so $\bar{\partial} \beta=0$ is equivalent to that $\nabla_{\bar{l}} \beta_{r}=0$ for any $l, r=1, \ldots, n$.

We also lower the index of torsion and denote it by $T_{i j \bar{k}}=T_{i j}^{s} g_{s \bar{k}}$. Note that $T^{\prime \prime}$ depends only on $J$ and it can be regarded as the Nijenhuis tensor of $J$, that is, $J$ is integrable if and only if $T^{\prime \prime}$ vanishes.

We denote by $\Omega$ the curvature of the Chern connection $\nabla$. We can regard $\Omega$ as a section of $\Lambda^{2} M \otimes T M$, $\Omega \in \Gamma\left(\Lambda^{2} M \otimes T M\right)$ and $\Omega$ splits in $\Omega=H+R+\bar{H}$ with

$$
\Omega^{(2,0)}=\left(\frac{1}{2} H_{k l i}^{j} \zeta^{k} \wedge \zeta^{l}\right), \quad \Omega^{(1,1)}=\left(R_{k \bar{l} i}^{j} \zeta^{k} \wedge \zeta^{\bar{l}}\right), \quad \Omega^{(0,2)}=\left(\frac{1}{2} H_{\bar{k} \bar{l} i}^{j} \zeta^{\bar{k}} \wedge \zeta^{\bar{l}}\right),
$$

where $R \in \Gamma\left(\Lambda^{1,1} M \otimes \Lambda^{1,1} M\right), H \in \Gamma\left(\Lambda^{2,0} M \otimes \Lambda^{1,1} M\right)$. The curvature form can be written by $\Omega_{j}^{i}=d \gamma_{j}^{i}+\gamma_{s}^{i} \wedge \gamma_{j}^{s}$. In terms of $Z_{r}$ 's, we have

$$
\begin{aligned}
& R_{i \overline{j k}}{ }^{r}=\Omega_{k}^{r}\left(Z_{i}, Z_{\bar{j}}\right)=Z_{i}\left(\Gamma_{\bar{j} k}^{r}\right)-Z_{\bar{j}}\left(\Gamma_{i k}^{r}\right)+\Gamma_{i s}^{r} \Gamma_{\bar{j} k}^{s}-\Gamma_{\bar{j} s}^{r} \Gamma_{i k}^{S}-B_{i j}^{s} \Gamma_{s k}^{r}+B_{\bar{j} i}^{\bar{s}} \Gamma_{\bar{s} k}^{r}=-R_{\bar{j} i k}{ }^{r}, \\
& H_{i j k}{ }^{r}=\Omega_{k}^{r}\left(Z_{i}, Z_{j}\right)=Z_{i}\left(\Gamma_{j k}^{r}\right)-Z_{j}\left(\Gamma_{i k}^{r}\right)+\Gamma_{i s}^{r} \Gamma_{j k}^{s}-\Gamma_{j s}^{r} \Gamma_{i k}^{s}-B_{i j}^{S} \Gamma_{s k}^{r}-B_{i j}^{\bar{s}} \Gamma_{\bar{s} k}^{r}=-H_{j i k}{ }^{r}, \\
& H_{\bar{i} \bar{j} k}{ }^{r}=\Omega_{k}^{r}\left(Z_{\bar{i}}, Z_{\bar{j}}\right)=Z_{\bar{i}}\left(\Gamma_{\bar{j} k}^{r}\right)-Z_{\bar{j}}\left(\Gamma_{\bar{i} k}^{r}\right)+\Gamma_{\bar{i} s}^{r} \Gamma_{\bar{j} k}^{S}-\Gamma_{\bar{j} s}^{r} \Gamma_{\bar{i} k}^{S}-B_{\bar{i} \bar{j}}^{S} \Gamma_{s k}^{r}-B_{\bar{i} j}^{\bar{s}} \Gamma_{\bar{s} k}^{r}=-H_{\bar{j} \bar{i} k}{ }^{r},
\end{aligned}
$$

and we deduce that with using $\Gamma_{k p}^{p}=Z_{k}(\log \operatorname{det} g)-B_{k \bar{p}}^{\bar{p}}$,

$$
\begin{gathered}
P_{i \bar{j}}=R_{i \bar{j} r}^{r}=-\left(Z_{i} Z_{\bar{j}}-\left[Z_{i}, Z_{\bar{j}}\right]^{(0,1)}\right)(\log \operatorname{det} g)+Z_{\bar{j}}\left(B_{i \bar{r}}^{\bar{r}}\right)+Z_{i}\left(B_{\bar{j} r}^{r}\right)+B_{i j}^{s} B_{s \bar{r}}^{\bar{r}}-B_{i j}^{\bar{s}} B_{\bar{s} r}^{r} \\
R_{i j}=H_{i j r}{ }^{r}=\left[Z_{i}, Z_{j}\right]^{(0,1)}(\log \operatorname{det} g)-Z_{i}\left(B_{j \bar{r}}^{\bar{r}}\right)+Z_{j}\left(B_{i \bar{r}}^{\bar{r}}\right)+B_{i j}^{s} B_{s \bar{r}}^{\bar{r}}+\overline{N_{i j}^{s}} B_{\bar{s} r}^{r}
\end{gathered}
$$

and

$$
R_{\overline{i j}}=H_{\bar{i} j r}{ }^{r}=-\left[Z_{\bar{i}}, Z_{\bar{j}}\right]^{(1,0)}(\log \operatorname{det} g)+Z_{\bar{i}}\left(B_{\overline{j r}}^{r}\right)-Z_{\bar{j}}\left(B_{\bar{i} r}^{r}\right)-N_{i j}^{S} B_{s \bar{r}}^{\bar{r}}-B_{i j}^{\bar{s}} B_{\bar{s} r}^{r} .
$$

The curvature $P$ is one of the Ricci-type curvatures of the Chern curvature. One has with an arbitrary $(1,0)$ frame $\left\{Z_{r}\right\}$ with respect to $g, P_{i \bar{j}}=g^{k \bar{l}} \Omega_{i \bar{j} k \bar{l}}$. We denote by $S$ one of the Ricci-type curvatures of the Chern curvature, which is locally given by $S_{i \bar{j}}=g^{k \bar{l}} \Omega_{k i \bar{j}}$. In the Kähler case, $S_{i \bar{j}}=P_{i \bar{j}}$ is the Ricci curvature.

We introduce the first Bianchi identity for the Chern curvature.

Lemma 2.2. (The first Bianchi identity for the Chern curvature) For any $X, Y, Z \in T^{\mathbb{C}} M$,

$$
\sum \Omega(X, Y) Z=\sum\left(T(T(X, Y), Z)+\nabla_{X} T(Y, Z)\right),
$$

where the sum is taken over all cyclic permutations.

This identity induces the following formulae:

$$
\begin{gathered}
R_{i \bar{j} k}{ }^{l}=R_{k \overline{j i}}{ }^{l}-T_{i k}^{\bar{r}} T_{\bar{r} \bar{j}}^{l}+\nabla_{\bar{j}} T_{k i}^{l}=R_{k \overline{j i}}{ }^{l}-B_{i k}^{\bar{r}} B_{\bar{r} \bar{j}}^{l}+\nabla_{\bar{j}} T_{k i}^{l}, \\
H_{i j k}{ }^{l}=T_{j i}^{\bar{r}} T_{\bar{r} \bar{l}}^{\bar{k}}+\nabla_{\bar{l}} T_{j i}^{\bar{k}}=-B_{j i}^{\bar{r}} T_{\bar{r} \bar{l}}^{\bar{k}}-\nabla_{\bar{l}} B_{j i}^{\bar{k}},
\end{gathered}
$$

where used that $R_{i j \bar{k} \bar{l}}=R_{\bar{i} \bar{j} k l}=H_{j \bar{l} i k}=H_{\bar{j} l i \bar{l} \bar{k}}=H_{\overline{l i j k}}=H_{l \bar{i} \bar{k} \bar{k}}=0$. 


\subsection{Complexified Riemannian curvature tensor}

We introduce the complexified Riemannian curvature tensor as in [17]. Let $(M, g, D)$ be a $2 n$-dimensional Riemannian manifold with Levi-Civita connection $D$. The tangent bundle of $M$ is denoted by $T^{\mathbb{R}} M$. The curvature tensor of $(M, g, D)$ is defined as

$$
R^{L}(X, Y, Z, W):=g\left(D_{Z} D_{W} X-D_{W} D_{Z} X-D_{[Z, W]} X, Y\right)
$$

for any $X, Y, Z, W \in T^{\mathbb{R}} M$. Let $T^{\mathbb{C}} M=T^{\mathbb{R}} M \otimes \mathbb{C}$ be the complexification of the tangent bundle $T^{\mathbb{R}} M$. We can extend the metric $g$, the Levi-Civita connection $D$ to $T^{\mathbb{C}} M$ in the $\mathbb{C}$-linear way. For any $a, b \in \mathbb{C}$ and $X, Y \in$ $T^{\mathbb{C}} M, g(a X, b Y):=a b \cdot g(X, Y)$. Hence for any $a, b, c, d \in \mathbb{C}$ and $X, Y, Z, W \in T^{\mathbb{C}} M, R^{L}(a X, b Y, c Z, d W)=$ abcd $\cdot R^{L}(X, Y, Z, W)$. Notice that the components of $\mathbb{C}$-linear complexified curvature tensor have the same properties as the components of the real curvature tensor. The components of the complexified curvature tensor $R_{i \bar{j} k \bar{l}}$ satisfies the following properties: $R_{i \bar{j} k \bar{l}}^{L}=-R_{\bar{j} i k \bar{l}}^{L}, R_{i \bar{j} k \bar{l}}^{L}=R_{k \bar{l} i \bar{j}}^{L}$ and the Bianchi identity: $R_{i \bar{j} k \bar{l}}^{L}+R_{i k \bar{l} \bar{j}}^{L}+$ $R_{i \bar{l} \bar{j} k}^{L}=0$.

\section{Almost Hermitian Kähler-like and G-Kähler-like structure}

We define the curvature with respect to the Levi-Civita connection $D$ in the following way for tangent vectors $X, Y, Z$ and $W$ :

$$
R^{L}(X, Y) Z=\left[D_{X}, D_{Y}\right] Z-D_{[X, Y]} Z, \quad R^{L}(X, Y, Z, W)=g\left(R^{L}(X, Y) Z, W\right) .
$$

An almost Hermitian manifold $(M, J, g)$ satisfying that (cf. [12], [13])

(i) $R^{L}(X, Y, Z, W)=R^{L}(X, Y, J Z, J W)$ for all vector fields $X, Y, Z, W$ is called an $A H_{1}$-manifold;

(ii) $R^{L}(X, Y, Z, W)=R^{L}(X, Y, J Z, J W)+R^{L}(X, J Y, Z, J W)+R^{L}(J X, Y, Z, J W)$ for all vector fields $X, Y, Z, W$ is called an $\mathrm{AH}_{2}$-manifold;

(iii) $R^{L}(X, Y, Z, W)=R^{L}(J X, J Y, J Z, J W)$ for all vector fields $X, Y, Z, W$ is called an $A H_{3}$-manifold.

(iv) $R^{L}(J X, J Y, Z, W)+R^{L}(J Y, J Z, X, W)+R^{L}(J Z, J X, Y, W)=0$ for all vector fields $X, Y, Z, W$ is called an $A H C$-manifold (cf. [13]).

Then $A H_{1} \subset A H_{2} \subset A H_{3}, A H_{1} \subset A H C \subset A H_{3}$, and $A H C \cap A H_{2}=A H_{1}$. Note that if an $A H_{1}$-manifold is almost Kähler, then it is Kähler (cf. [12, Theorem 5.1]). Furthermore, it is known that an almost Kählerian or a nearly Kählerian $A H C$-manifold $M^{2 n}$ is Kählerian (cf. [13, Lemma 10.3]). It is well-known that a nearly Kähler manifold automatically satisfy the $\mathrm{AH}_{3}$-condition (cf. [11]).

The curvature $R^{L}$ of the Levi-Civita connection $D$ satisfies the first Bianchi identity:

$$
\text { (1Bnc) } \sum_{\sigma \in \mathfrak{G}} R^{L}(\sigma x, \sigma y) \sigma z=0,
$$

where the sum is taken over all cyclic permutations. The curvature $\Omega^{(1,1)}=R$ of the Chern connection $\nabla$ satisfies

$$
\text { (Cplx) } R(x, y, z, w)=R(x, y, J z, J w)=R(J x, J y, z, w) .
$$

We define the Kähler-likeness in the way of [3, Definition 4] as follows.

Definition 3.1. Let $M$ be an almost complex manifold endowed with an almost Hermitian structure. Let $\nabla$ be a metric connection on it. We say that the curvature of $\nabla$ is Kähler-like if it satisfies (1Bnc) and (Cplx).

We see the following equivalences which are similar to the ones in [3, Remark 5].

Lemma 3.1. (i) An almost Hermitian structure is Kähler-like in the sense of Definition 1.2 if and only if the curvature $\Omega^{(1,1)}=R$ of the Chern connection is Kähler-like in the sense of Definition 3.1. 
(ii) An almost Hermitian structure is G-Kähler-like in the sense of Definition 1.2 if and only if the curvature $R^{L}$ of the Levi-Civita connection is Kähler-like in the sense of Definition 3.1.

Proof. (i) Assume that the curvature $\Omega^{(1,1)}=R$ of the Chern connection $\nabla$ is Kähler-like in the sense of Definition 1.6. By using (1Bnc), we obtain

$$
R(X, \bar{Y}, Z, \bar{W})=-R(\bar{Y}, Z, X, \bar{W})-R(Z, X, \bar{Y}, \bar{W})=R(Z, \bar{Y}, X, \bar{W})
$$

for any $(1,0)$-tangent vector fields $X, Y, Z$ and $W$. On the other hand, assume that the almost Hermitian structure is Kähler-like in the sense of Definition 1.2. Since the Chern connection satisfies (Cplx), it suffices to show (1Bnc) for

$$
(x, y, z, w) \in\{(\bar{X}, Y, Z, \bar{W}),(X, \bar{Y}, Z, \bar{W}),(X, Y, \bar{Z}, \bar{W})\},
$$

where $X, Y, Z$ and $W$ are $(1,0)$-tangent vector fields. For instance, in the first case, we have

$$
R(\bar{X}, Y, Z, \bar{W})+R(Y, Z, \bar{X}, \bar{W})+R(Z, \bar{X}, Y, \bar{W})=-R(Y, \bar{X}, Z, \bar{W})+R(Z, \bar{X}, Y, \bar{W})=0 .
$$

(ii) Assume that the curvature $R^{L}$ of the Levi-Civita connection $D$ is Kähler-like in the sense of Definition 2.1. By using (Cplx), we obtain for any $(1,0)$-tangent vector fields $X, Y, Z$, and $W$,

$$
R^{L}(X, Y, \bar{Z}, \bar{W})=R^{L}(X, Y, J \bar{Z}, J \bar{W})=-R^{L}(X, Y, \bar{Z}, \bar{W})
$$

for $(1,0)$-tangent vector fields $X, Y, Z$ and $W$. Hence we get $R^{L}(X, Y, \bar{Z}, \bar{W})=0$. Furthermore, by applying $R^{L}(x, y, z, w)=R^{L}(z, w, x, y)$,

$$
R^{L}(X, Y, Z, \bar{W})=R^{L}(Z, \bar{W}, X, Y)=R^{L}(Z, \bar{W}, J X, J Y)=-R^{L}(X, Y, Z, \bar{W}) .
$$

Therefore, we get $R^{L}(X, Y, Z, \bar{W})=0$ for all $(1,0)$-tangent vector fields $X, Y, Z$ and $W$.

On the other hand, assume that the almost Hermitian structure is G-Kähler-like in the sense of Definition 1.2. For any (1, 0)-tangent vector fields $X, Y, Z$, and $W, R^{L}(X, Y, Z, W)=0$ (cf. [10]). Since the Levi-Civita connection satisfies (1Bnc), it suffices to show $R^{L}(x, y, z, w)=R^{L}(x, y, J z, J w)$. Then the terms of curvatures are reduced as follows: $R^{L}(\bar{X}, Y, Z, \bar{W}), R^{L}(X, \bar{Y}, Z, \bar{W})$, and $R^{L}(X, Y, Z, \bar{W})=R^{L}(X, Y, \bar{Z}, \bar{W})=R^{L}(\bar{X}, Y, \bar{Z}, \bar{W})=$ $R^{L}(X, \bar{Y}, \bar{Z}, \bar{W})=R^{L}(\bar{X}, \bar{Y}, Z, \bar{W})=R^{L}(\bar{X}, \bar{Y}, \bar{Z}, \bar{W})=0$. Hence in this case, the condition (Cplx) holds.

Remark 3.1. Since we have the symmetry $R^{L}(x, y, z, w)=R^{L}(z, w, x, y)$, and since the Levi-Civita connection always satisfies (1Bnc), the $A H_{1}$-condition is equivalent to that the curvature $R^{L}$ of the Levi-Civita connection is Kähler-like in the sense of Definition 3.1. From Lemma 3.1 (ii), consequently, the G-Kähler-like condition in Definition 1.2 is equivalent to the $A H_{1}$-condition. Recall that if an $A H_{1}$-manifold is almost Kähler, then it must be Kähler. Combining with the last statement in Proposition 1.3, we have the result that a real 4-dimensional compact Kähler-like and G-Kähler-like almost Hermitian manifold is Kähler as in Corollary 1.1.

Lemma 3.2. Let $(M, J, g)$ be a G-Kähler-like almost Hermitian manifold. If $R_{i j \bar{j} \bar{l}}=R_{i j k \bar{l}}^{L}$ for all $i, j, k, l=$ $1, \ldots, n$, then the metric $g$ is Kähler-like in the sense of Definition 1.2.

Proof. Since the curvature $\Omega^{(1,1)}=R$ satisfies (Cplx), by showing $R$ satisfies (1Bnc), then we have that $R$ is Kähler-like in the sense of Definition 2.1. From Lemma 3.1 ( $i$ ), then we conclude that $g$ is Kähler-like in the sense of Definition 1.2.

We see that $R$ satisfies (1Bnc). Since we have assumed that $R_{i \bar{j} k \bar{l}}=R_{i j \bar{k} \bar{l}}^{L}$ for all $i, j, k, l=1, \ldots, n$ and since we have that $R_{k i \bar{j}}=0$, we obtain that

$$
\begin{aligned}
0 & =R_{i \overline{j k l}}^{L}+R_{j k i \bar{l}}^{L}+R_{k i \bar{l} \bar{l}}^{L} \\
& =R_{i \bar{j} k \bar{l}}^{L}-R_{k j \bar{i} \bar{l}}^{L} \\
& =R_{i j \bar{j} \bar{l}}-R_{k j \bar{l}} \\
& =R_{i \bar{j} k \bar{l}}+R_{\bar{j} k i \bar{l}}+R_{k i \bar{l} \bar{l}},
\end{aligned}
$$

where we used that $R_{k i \bar{j} l}^{L}=0$ since we have assumed that $(M, J, g)$ is G-Kähler-like and also we used that $R_{\bar{j} k i \bar{l}}=-R_{k j \bar{j} \bar{i}}$. Therefore, $R$ satisfies (1Bnc). 
By applying Lemma 3.2, since the G-Kähler-likeness and that $R_{i j \bar{k} \bar{l}}=R_{i j k \bar{l}}^{L}$ for all $i, j, k, l=1, \ldots, n$ imply the Kähler-likeness, if the manifold is compact, we obtain the following corollary by applying Theorem 1.1.

Corollary 3.1. Let $\left(M^{2 n}, J, g\right)$ be a compact G-Kähler-like almost balanced manifold with $n \geq 2$. Assume that $R_{i \bar{j} k \bar{l}}=R_{i \bar{j} k \bar{l}}^{L}$ for all $i, j, k, l=1, \ldots, n$. Then $g$ is Kähler.

By applying Corollary 1.2, we have the following result.

Corollary 3.2. Let $\left(M^{2 n}, J, g\right)$ be a G-Kähler-like quasi-Kähler manifold with $n \geq 2$. Assume that $R_{i \bar{j} k \bar{l}}=R_{i \bar{j} k \bar{l}}^{L}$ for all $i, j, k, l=1, \ldots, n$ and $B_{i j}^{\lambda} B_{\lambda j}^{\bar{i}} \geq 0$. Then $g$ is Kähler.

Next, we introduce the following result for $A H_{1}$-manifolds.

Lemma 3.3. (cf. [13, Theorem 9.2]) If a $2 n$-dimensional $A H_{1}$-manifold $M^{2 n}$ for $n>1$ has constant general sectional curvature $K$ at a point, then $M^{2 n}$ is locally flat.

From Lemma 3.1 (ii) and Lemma 3.3, we may say that if a G-Kähler-like manifold has constant general sectional curvature $K$ at a point, then the manifold is locally flat.

Here, we define $w_{r}:=T_{r i}^{i}$ and the torsion (1,0)-form $\eta:=T_{i r}^{i} \zeta^{r}=-w_{r} \zeta^{r}$ (cf. [23]), where $T=\left(T^{i}\right)$ is the torsion of the Chern connection $\nabla$. We also define an almost Gauduchon metric as follows.

Definition 3.2. Let $\left(M^{2 n}, J\right)$ be an almost complex manifold. A metric $g$ is called an almost Gauduchon metric on $M$ if $g$ is an almost Hermitian metric whose associated real $(1,1)$-form $\omega=\sqrt{-1} g_{i j} \zeta^{i} \wedge \zeta^{j}$ satisfies $d^{\star}\left(J d^{\star} \omega\right)=$ 0 , where $d^{*}$ is the adjoint of $d$ with respect to $g$, which is equivalent to $d\left(J d\left(\omega^{n-1}\right)\right)=0$. When an almost Hermitian metric $g$ is almost Gauduchon, the triple $\left(M^{2 n}, J, g\right)$ will be called an almost Gauduchon manifold.

One has the following well-known result.

Proposition 3.1. (cf. [8]) Let $\left(M^{2 n}, J, \omega\right), n \geq 2$, be a compact almost Hermitian manifold. Then there exists a smooth function $u$, unique up to addition of a constant, such that the conformal almost Hermitian metric $e^{u} \omega$ is almost Gauduchon.

We shall show that the torsion $(1,0)$-form $\eta$ satisfies that $\operatorname{Re}(\bar{\partial} \eta)=0$ if and only if $g$ is almost Gauduchon in Lemma 4.4. By applying Theorem 1.2, then we obtain the following result.

Corollary 3.3. Let $\left(M^{2 n}, J, g\right)$ be a G-Kähler-like almost Gauduchon manifold with $n \geq 2$. Assume that $B_{i j}^{\lambda} B_{\lambda j}^{\bar{i}} \geq$ 0 . Then the metric $g$ is almost balanced and the almost complex structure $J$ is integrable (i.e. $g$ is balanced).

We consider the case of quasi-Kähler manifolds. The following lemma can be obtained easily to see the expression of (2.30).

Lemma 3.4. (cf. [20, Lemma 2.4]) An almost Hermitian manifold $\left(M^{2 n}, g, J\right)$ is quasi-Kähler if and only if $T_{i j}^{k}=0$ for all $i, j$ and $k$ when a local unitary $(1,0)$-frame is fixed.

By applying Lemma 3.4, we have the following corollary.

Corollary 3.4. A G-Kähler-like quasi-Kähler manifold with $B_{i j}^{\lambda} B_{\lambda j}^{\bar{i}} \geq 0$ is Kähler.

We see that if the metric is pluriclosed and balanced at the same time, it must be Kähler as follows.

Proposition 3.2. ([19, Proposition 1.1]) Let $(M, J, g)$ be a Hermitian manifold with the associated real $(1,1)$ form $\omega$. If $\omega$ is pluriclosed and balanced, then $\omega$ is Kähler. 
We define an almost pluriclosed metric on almost complex manifolds.

Definition 3.3. ([14, Definition 1.1]) Let $(M, J)$ be an almost complex manifold. A metric $g$ is called an almost pluriclosed metric on $M$ if $g$ is an almost Hermitian metric whose associated real $(1,1)$-form (,which is called the fundamental $(1,1)$-form $\omega=g(J \cdot, \cdot)$ of the almost Hermitian metric $g,) \omega=\sqrt{-1} g_{i \bar{j}} \zeta^{i} \wedge \zeta^{\bar{j}}$ satisfies $\partial \bar{\partial} \omega=0$.

Then by combining Corollary 1.2 and Proposition 3.2, we obtain the following result.

Corollary 3.5. Let $\left(M^{2 n}, J, g\right)$ be a Kähler-like and G-Kähler-like almost Hermitian manifold with $n \geq 2$. Assume that $B_{\bar{i} j} B_{\lambda j}^{\bar{i}} \geq 0$ and the metric $g$ is almost pluriclosed. Then $g$ is Kähler.

Obviously, when the metric $g$ is Kähler, one has $R=R^{L}$. Yang and Zheng have shown the following result in the Hermitian case.

Proposition 3.3. ([23, Theorem 1.1]) Given a Hermitian manifold $\left(M^{n}, J, g\right)$, if its Riemannian curvature tensor $R^{L}$ and its Hermitian curvature tensor $R$ are equal, then $g$ is Kähler.

We study the case $R_{i \bar{j} k \bar{l}}^{L}$ and $R_{i \bar{j} k \bar{l}}$ are equal in the almost Hermitian geometry.

Theorem 3.1. Let $\left(M^{2 n}, J, g\right)$ be a G-Kähler-like almost Hermitian manifold with $n \geq 2$. Assume that $R_{i j \bar{k} \bar{l}}=$ $R_{i j k l}^{L}$ for all $i, j, k, l=1, \ldots, n$ and $B_{i j}^{\lambda} B_{\lambda j}^{\bar{i}} \geq 0$. Then $g$ is Kähler.

By applying Corollary 3.5 (or Theorem 1.1) and Proposition 1.3, we obtain the following.

Corollary 3.6. Let $\left(M^{2 n}, J, g\right)$ be a compact G-Kähler-like almost Hermitian manifold with $n \geq 2$. Assume that $R_{i j k \bar{l}}=R_{i j k \bar{l}}^{L}$ for all $i, j, k, l=1, \ldots, n$. If $M^{2 n}$ admits a positive $\partial \bar{\partial}$-closed $(n-2, n-2)$-form $\chi$, then $g$ is Kähler. In particular, if $M^{2 n}$ is compact, G-Kähler-like, and $R_{i j \bar{k} \bar{l}}=R_{i j \bar{j} \mathrm{l}}^{L}$ for all $i, j, k, l=1, \ldots, n$ and $\partial \bar{\partial}\left(\omega^{n-2}\right)=0$, then $g$ is Kähler.

\section{Proofs of Theorem 1.1 and 1.2}

First, we show the following identity.

Lemma 4.1. Let $(M, J, g)$ be an almost Hermitian manifold. Let $\left\{Z_{r}\right\}$ be a local $(1,0)$-unitary frame with respect to $g$. Then one has

$$
R_{i \bar{j} k \bar{l}}=g\left(\nabla_{i} \nabla_{\bar{j}} Z_{k}-\nabla_{\bar{j}} \nabla_{i} Z_{k}-\nabla_{\left[Z_{i}, Z_{j}\right.} Z_{k}, Z_{\bar{l}}\right) .
$$

Proof. By using a local $g$-unitary $(1,0)$-frame $\left\{Z_{r}\right\}$, we obtain the following computation:

$$
\begin{aligned}
& g\left(\nabla_{i} \nabla_{\bar{j}} Z_{k}-\nabla_{\bar{j}} \nabla_{i} Z_{k}-\nabla_{\left[Z_{i}, Z_{\bar{j}}\right]} Z_{k}, Z_{\bar{l}}\right) \\
= & Z_{i}\left(g\left(\nabla_{\bar{j}} Z_{k}, Z_{\bar{l}}\right)\right)-g\left(\nabla_{\bar{j}} Z_{k}, \nabla_{i} Z_{\bar{l}}\right)-Z_{\bar{j}}\left(g\left(\nabla_{i} Z_{k}, Z_{\bar{l}}\right)+g\left(\nabla_{i} Z_{k}, \nabla_{\bar{j}} Z_{\bar{l}}\right)\right. \\
& -B_{i \bar{j}}^{r} g\left(\nabla_{r} Z_{k}, Z_{\bar{l}}\right)-B_{i \bar{j}}^{\bar{r}} g\left(\nabla_{\bar{r}} Z_{k}, Z_{\bar{l}}\right) \\
= & Z_{i}\left(\Gamma_{\bar{j} k}^{s} g_{s \bar{l}}\right)-\Gamma_{\bar{j} k}^{s} \Gamma_{i \bar{l}}^{\bar{r}} g_{s \bar{r}}-Z_{\bar{j}}\left(\Gamma_{i k}^{s} g_{s \bar{l}}\right)+\Gamma_{i k}^{s} \Gamma_{\bar{j} l}^{\bar{r}} g_{s \bar{r}}-B_{i \bar{j}}^{s} \Gamma_{s k}^{r} g_{r \bar{l}}-B_{i \bar{j}}^{\bar{r}} \Gamma_{\bar{r} k}^{s} g_{s \bar{l}} \\
= & Z_{i}\left(\Gamma_{\bar{j} k}^{l}\right)-Z_{\bar{j}}\left(\Gamma_{i k}^{l}\right)+\Gamma_{i s}^{l} \Gamma_{\bar{j} k}^{s}-\Gamma_{\bar{j} s}^{l} \Gamma_{i k}^{s}-B_{i j}^{s} \Gamma_{s k}^{l}-B_{i j}^{\bar{s}} \Gamma_{\bar{s} k}^{l} \\
= & R_{i \bar{j} k}{ }^{s} g_{s \bar{l}} \\
= & R_{i \bar{j} \bar{l} \bar{l}} .
\end{aligned}
$$

Therefore we obtain the desired formula. 
Let $\left(M^{2 n}, J, g\right)$ be an almost Hermitian manifold and let $\nabla$ be the Chern connection on $M$. Let $\left\{Z_{r}\right\}$ be a local $(1,0)$-frame with respect to $g$ around a fixed point $p \in M$ such that $\nabla Z_{i}(p)=0$ and $g_{i \bar{j}}(p)=\delta_{i j}$, and let $\left\{\zeta^{r}\right\}$ be the associated coframe. The existence of such frames has been proven in [25].

In order to prove Theorem 1.1, we introduce the following lemma. By applying the first Bianchi identity for the Chern curvature (2.40), we obtain the formula as follows.

Lemma 4.2. ([15, Lemma 3.1]) Let $\left(M^{2 n}, J, g\right)$ is a Kähler-like almost Hermitian manifold. Then we have

$$
(\bar{\nabla} w)_{k \bar{j}}=(\nabla \bar{w})_{k \bar{j}}=B_{i k}^{\bar{q}} B_{\bar{q} \bar{j}}^{i} .
$$

Recall the torsion (1,0)-form $\eta=T_{i r}^{i} \zeta^{r}=-w_{r} \zeta^{r}$, where $w_{r}=T_{r i}^{i}$, as we defined in the previous section. Notice that when a Hermitian manifold is Kähler-like, we obtain that $\bar{\partial} \eta=0$ as we see below.

Proposition 4.1. ([23, Lemma 6]) If a Hermitian manifold $\left(M^{n}, J, g\right)$ is Kähler-like, then its torsion 1-form $\eta$ is holomorphic. The converse of this is also true if $n=2$.

From the equation (2.31), we have $\bar{\partial} \eta=\nabla_{\bar{l}} w_{r} \zeta^{r} \wedge \zeta^{\bar{l}}$. Hence $\bar{\partial} \eta=0$ is equivalent to that $\nabla_{\bar{l}} w_{r}=0$ for any $l, r=1, \ldots, n$. By applying (4.3), we can obtain the same result as in the first statement of Proposition 4.1, since we have $\nabla_{\bar{l}} w_{r}=B_{i r}^{\bar{q}} B_{\bar{q} \bar{l}}^{i}=N_{i r}^{\bar{q}} N_{\bar{q} \bar{l}}^{i}=0$ for any $r, l=1, \ldots, n$ on a Kähler-like Hermitian manifold.

Lemma 4.3. On an almost Hermitian manifold $(M, J, g)$, the metric $g$ is almost balanced if and only if the torsion (1,0)-form $\eta$ satisfies $\eta=0$.

Proof. As we see in section 2, we have $\partial \omega=\frac{\sqrt{-1}}{2} T_{i j \bar{k}} \zeta^{i} \wedge \zeta^{j} \wedge \zeta^{\bar{k}}$. Then a direct calculation shows that

$$
\partial \omega^{n-1}=(n-1)\left(\frac{\partial \omega \wedge \omega^{n-2}}{\omega^{n-1}}\right) \wedge \omega^{n-1}=-\eta \wedge \omega^{n-1},
$$

where we used that $\eta=-w_{i} \zeta^{i}=-(n-1) \frac{\partial \omega \wedge \omega^{n-2}}{\omega^{n-1}}$. Similarly, we obtain that

$$
\bar{\partial} \omega^{n-1}=(n-1)\left(\frac{\bar{\partial} \omega \wedge \omega^{n-2}}{\omega^{n-1}}\right) \wedge \omega^{n-1}=-\bar{\eta} \wedge \omega^{n-1},
$$

since we have $\bar{\partial} \omega=\frac{\sqrt{-1}}{2} T_{\overline{j i k}} \zeta^{k} \wedge \zeta^{\bar{i}} \wedge \zeta^{\bar{j}}$ and $\bar{\eta}=-w_{\bar{i}} \zeta^{\bar{i}}=-(n-1) \frac{\bar{\partial} \omega \wedge \omega^{n-2}}{\omega^{n-1}}$.

Recall that the metric $g$ is said to be almost balanced if $\omega^{n-1}$ is closed. Since we have $A \omega^{n-1}=\bar{A} \omega^{n-1}=0$, we get $d \omega^{n-1}=\partial \omega^{n-1}+\bar{\partial} \omega^{n-1}$. Therefore, these identities (4.4), (4.5) show that $g$ is almost balanced if and only if $\eta=0$.

From Lemma 4.3, we obtain the following result by applying Proposition 1.2.

Proposition 4.2. On a compact Kähler-like almost Hermitian manifold $(M, J, g)$, the torsion $(1,0)$-form $\eta$ satisfies $\eta=0$ if and only if $B_{i k}^{\bar{q}} B_{\bar{q} \bar{l}}^{i}=0$ for all $k, l=1, \ldots, n$.

Let $\left(M^{2 n}, J, g\right)$ be an almost Hermitian manifold. We say that $g$ is almost Gauduchon if the associated real $(1,1)$-form $\omega$ with respect to $g$ satisfies $d^{\star}\left(J d^{\star} \omega\right)=0$, where $d^{\star}$ is the adjoint of $d$ with respect to $g$, which is equivalent to $d\left(J d\left(\omega^{n-1}\right)\right)=0$. We have the following equivalence.

Lemma 4.4. Let $\left(M^{2 n}, J, g\right)$ be an almost Hermitian manifold with $n \geq 2$. The torsion $(1,0)$-form $\eta$ satisfies that $\operatorname{Re}(\bar{\partial} \eta)=0$ if and only if $g$ is almost Gauduchon.

Proof. Note that we extend the action of $J$ to $p$-forms $\alpha$ by

$$
(J \alpha)\left(Z_{1}, \ldots, Z_{p}\right):=(-1)^{p} \alpha\left(J Z_{1}, \ldots, J Z_{p}\right) .
$$


Since we have $d \omega^{n-1}=(A+\partial+\bar{\partial}+\bar{A}) \omega^{n-1}=(\partial+\bar{\partial}) \omega^{n-1}=-(\eta+\bar{\eta}) \wedge \omega^{n-1}$, for any $(1,0)$-vector fields $Z_{1}, \ldots, Z_{n}$, we have

$$
\begin{aligned}
J d\left(\omega^{n-1}\right)\left(Z_{1}, \ldots, Z_{n}\right) & =(-1)^{n} d\left(\omega^{n-1}\right)\left(J Z_{1}, \ldots, J Z_{n}\right) \\
& =(-\sqrt{-1})^{n} d\left(\omega^{n-1}\right)\left(Z_{1}, \ldots, Z_{n}\right) \\
& =-(-\sqrt{-1})^{n}(\eta+\bar{\eta}) \wedge \omega^{n-1}\left(Z_{1}, \ldots, Z_{n}\right) .
\end{aligned}
$$

Hence, we compute

$$
\begin{aligned}
& d\left(J d\left(\omega^{n-1}\right)\right)\left(Z_{1}, \ldots, Z_{n}\right) \\
= & -(-\sqrt{-1})^{n} d\left((\eta+\bar{\eta}) \wedge \omega^{n-1}\right)\left(Z_{1}, \ldots, Z_{n}\right) \\
= & -(-\sqrt{-1})^{n}\left\{d(\eta+\bar{\eta}) \wedge \omega^{n-1}\left(Z_{1}, \ldots, Z_{n}\right)-(\eta+\bar{\eta}) \wedge d \omega^{n-1}\left(Z_{1}, \ldots, Z_{n}\right)\right\} \\
= & -(-\sqrt{-1})^{n}\left\{(\bar{\partial} \eta+\partial \bar{\eta}) \wedge \omega^{n-1}\left(Z_{1}, \ldots, Z_{n}\right)+(\eta+\bar{\eta}) \wedge(\eta+\bar{\eta}) \wedge \omega^{n-1}\left(Z_{1}, \ldots, Z_{n}\right)\right\} \\
= & -(-\sqrt{-1})^{n}\left\{(\bar{\partial} \eta+\partial \bar{\eta}) \wedge \omega^{n-1}\left(Z_{1}, \ldots, Z_{n}\right)+\eta \wedge \bar{\eta} \wedge \omega^{n-1}\left(Z_{1}, \ldots, Z_{n}\right)\right. \\
& \left.+\bar{\eta} \wedge \eta \wedge \omega^{n-1}\left(Z_{1}, \ldots, Z_{n}\right)\right\} \\
= & -(-\sqrt{-1})^{n}(\bar{\partial} \eta+\partial \bar{\eta}) \wedge \omega^{n-1}\left(Z_{1}, \ldots, Z_{n}\right) \\
= & -(-\sqrt{-1})^{n}\left(2 \operatorname{Re}(\bar{\partial} \eta) \wedge \omega^{n-1}\right)\left(Z_{1}, \ldots, Z_{n}\right),
\end{aligned}
$$

where we have used that $A \eta \wedge \omega^{n-1}=\bar{A} \eta \wedge \omega^{n-1}=0$ and $\partial \eta \wedge \omega^{n-1}=0$.

Proof of Theorem 1.1. Suppose that $\left(M^{2 n}, J, g\right)$ be a compact Kähler-like and G-Kähler-like almost balanced manifold with $n \geq 2$. Let $D$ be the Levi-Civita connection and $R^{L}$ denotes its curvature tensor with respect to $g$. Recall the definition of curvature operator: for type $(1,0)$ tangent vectors $X, Y, Z$,

$$
R^{L}(X, Y) Z:=D_{X} D_{Y} Z-D_{Y} D_{X} Z-D_{[X, Y]} Z \text {. }
$$

The curvature tensor is defined for type $(1,0)$ tangent vectors $X, Y, Z$ and $W$ as

$$
R^{L}(X, Y, Z, W):=g\left(R^{L}(Z, W) X, Y\right) .
$$

The torsion $T$ with respect to the Chern connection $\nabla$ is a vector-valued 2-form given by $T(X, Y)=\nabla_{X} Y-$ $\nabla_{Y} X-[X, Y]$ satisfying $T(X, \bar{Y})=0$ for any type $(1,0)$ tangent vectors $X, Y$. Recall the following comparison of the Levi-Civita connection and the Chern connection on almost Hermitian manifolds.

Lemma 4.5. (cf. [24, Lemma 3.1])

$$
g\left(D_{Y} X, Z\right)=g\left(\nabla_{Y} X, Z\right)+\frac{1}{2}(g(T(X, Y), Z)+g(T(Y, Z), X)-g(T(Z, X), Y))
$$

for any type $(1,0)$ tangent vectors $X, Y, Z$.

For an arbitrary chosen fixed point $p \in M$, let $\left\{Z_{r}\right\}$ be a local $(1,0)$-frame at $p$ such that $\nabla Z_{r}(p)=0$ and $g_{i j}(p)=\delta_{i j}$. By applying (4.11), we compute at $p$,

$$
\begin{gathered}
g\left(D_{Z_{\bar{\lambda}}} Z_{i}, Z_{\mu}\right)=-\frac{1}{2} g\left(T\left(Z_{\mu}, Z_{i}\right), Z_{\bar{\lambda}}\right)=-\frac{1}{2} T_{\mu i}^{\lambda}=\frac{1}{2} T_{i \mu}^{\lambda}, \\
g\left(D_{Z_{\bar{\lambda}}} Z_{i}, Z_{\bar{\mu}}\right)=\frac{1}{2} g\left(T\left(Z_{\bar{\lambda}}, Z_{\bar{\mu}}\right), Z_{i}\right)=\frac{1}{2} T_{\bar{\lambda} \bar{\mu}}^{\bar{i}}, \\
g\left(D_{\lambda} Z_{i}, Z_{\bar{\mu}}\right)=\frac{1}{2} g\left(T\left(Z_{i}, Z_{\lambda}\right), Z_{\bar{\mu}}\right)=\frac{1}{2} T_{i \lambda}^{\mu}, \\
g\left(D_{\lambda} Z_{i}, Z_{\mu}\right)=\frac{1}{2}\left(g\left(T\left(Z_{i}, Z_{\lambda}\right), Z_{\mu}\right)+g\left(T\left(Z_{\lambda}, Z_{\mu}\right), Z_{i}\right)-g\left(T\left(Z_{\mu}, Z_{i}\right), Z_{\lambda}\right)\right)=\frac{1}{2}\left(T_{i \lambda}^{\bar{\mu}}+T_{\lambda \mu}^{\bar{i}}-T_{\mu i}^{\bar{\lambda}}\right) .
\end{gathered}
$$


Hence, we obtain

and

$$
D_{Z_{\bar{\lambda}}} Z_{i}(p)=\frac{1}{2} T_{i \mu}^{\lambda}(p) Z_{\bar{\mu}}+\frac{1}{2} T_{\bar{\lambda} \bar{\mu}}^{\bar{i}}(p) Z_{\mu}
$$

$$
D_{Z_{\lambda}} Z_{i}(p)=\frac{1}{2} T_{i \lambda}^{\mu}(p) Z_{\mu}+\frac{1}{2}\left(T_{i \lambda}^{\bar{\mu}}+T_{\lambda \mu}^{\bar{i}}-T_{\mu i}^{\bar{\lambda}}\right)(p) Z_{\bar{\mu}} .
$$

Using (4.11) and (4.16), (4.17), we compute at $p$,

$$
\begin{aligned}
g\left(D_{Z_{\bar{k}}} D_{Z_{\bar{l}}} Z_{i}, Z_{j}\right) & =g\left(\nabla_{Z_{\bar{k}}} D_{Z_{\bar{l}}} Z_{i}, Z_{j}\right)+\frac{1}{2}\left(g\left(T\left(D_{Z_{\bar{l}}} Z_{i}, Z_{\bar{k}}\right), Z_{j}\right)-g\left(T\left(Z_{j}, D_{Z_{\bar{l}}} Z_{i}\right), Z_{\bar{k}}\right)\right) \\
& =Z_{\bar{k}}\left(g\left(D_{Z_{\bar{l}}} Z_{i}, Z_{j}\right)\right)+\frac{1}{4} T_{i \lambda}^{l} T_{\bar{\lambda} \bar{k}}^{\bar{j}}-\frac{1}{4} T_{\bar{l} \bar{\lambda}}^{\bar{i}} T_{j \lambda}^{k} \\
& =Z_{\bar{k}}\left(g\left(\nabla_{\bar{l}} Z_{i}, Z_{j}\right)-\frac{1}{2} g\left(T\left(Z_{j}, Z_{i}\right), Z_{\bar{l}}\right)\right)+\frac{1}{4} T_{i \lambda}^{l} T_{\bar{\lambda} \bar{k}}^{\bar{j}}-\frac{1}{4} T_{\bar{l} \bar{\lambda}}^{\bar{i}} T_{j \lambda}^{k} \\
& =g\left(\nabla_{\bar{k}} \nabla_{\bar{l}} Z_{i}, Z_{j}\right)-\frac{1}{2} \nabla_{\bar{k}} T_{j i}^{l}+\frac{1}{4} T_{i \lambda}^{l} T_{\bar{\lambda} \bar{k}}^{\bar{j}}-\frac{1}{4} T_{\bar{l} \bar{\lambda}}^{\bar{i}} T_{j \lambda}^{k} .
\end{aligned}
$$

Similarly, we have at $p$,

$$
g\left(D_{Z_{\bar{l}}} D_{Z_{\bar{k}}} Z_{i}, Z_{j}\right)=g\left(\nabla_{\bar{l}} \nabla_{\bar{k}} Z_{i}, Z_{j}\right)-\frac{1}{2} \nabla_{\bar{l}} T_{j i}^{k}+\frac{1}{4} T_{i \lambda}^{k} T_{\bar{\lambda} \bar{l}}^{\bar{j}}-\frac{1}{4} T_{\bar{k} \bar{\lambda}}^{\bar{i}} T_{j \lambda}^{l} .
$$

Since we have

$$
\begin{aligned}
{\left[Z_{\bar{k}}, Z_{\bar{l}}\right](p) } & =\nabla_{\bar{k}} Z_{\bar{l}}(p)-\nabla_{\bar{l}} Z_{\bar{k}}(p)-T\left(Z_{\bar{k}}, Z_{\bar{l}}\right)(p) \\
& =-T_{\bar{k} \bar{l}}^{\lambda}(p) Z_{\lambda}-T_{\bar{k} \bar{l}}^{\bar{\lambda}}(p) Z_{\bar{\lambda}},
\end{aligned}
$$

we then get at $p$,

$$
\begin{aligned}
g\left(D_{\left[Z_{\bar{k}}, Z_{\bar{l}}\right]} Z_{i}, Z_{j}\right) & =-T_{\bar{k} \bar{l}}^{\lambda} g\left(D_{Z_{\lambda}} Z_{i}, Z_{j}\right)-T_{\bar{k} \bar{l}}^{\bar{\lambda}} g\left(D_{Z_{\bar{\lambda}}} Z_{i}, Z_{j}\right) \\
& =-\frac{1}{2} T_{\bar{k} \bar{l}}^{\lambda}\left(T_{i \lambda}^{\bar{j}}+T_{\lambda j}^{\bar{i}}-T_{j i}^{\bar{\lambda}}\right)-\frac{1}{2} T_{\bar{k} \bar{l}}^{\bar{\lambda}} T_{i j}^{\lambda} .
\end{aligned}
$$

Hence, we have at $p$,

$$
\begin{aligned}
R_{i j \bar{k} \bar{l}}^{L}= & g\left(D_{Z_{\bar{k}}} D_{Z_{\bar{l}}} Z_{i}-D_{Z_{\bar{l}}} D_{Z_{\bar{k}}} Z_{i}-D_{\left[Z_{\bar{k}}, Z_{\bar{l}}\right]} Z_{i}, Z_{j}\right) \\
= & g\left(\left(\nabla_{\bar{k}} \nabla_{\bar{l}}-\nabla_{\bar{l}} \nabla_{\bar{k}}-\nabla_{\left[Z_{\bar{k}}, Z_{\bar{l}}\right]}\right) Z_{i}, Z_{j}\right)-\frac{1}{2} \nabla_{\bar{k}} T_{j i}^{l}+\frac{1}{2} \nabla_{\bar{l}} T_{j i}^{k}+\frac{1}{4} T_{i \lambda}^{l} T_{\bar{\lambda} \bar{k}}^{\bar{j}} \\
& -\frac{1}{4} T_{\bar{l} \bar{\lambda}}^{\bar{i}} T_{j \lambda}^{k}-\frac{1}{4} T_{i \lambda}^{k} T_{\bar{\lambda} \bar{l}}^{\bar{j}}+\frac{1}{4} T_{\bar{k} \bar{\lambda}}^{\bar{i}} T_{j \lambda}^{l}+\frac{1}{2} T_{\bar{k} \bar{l}}^{\lambda}\left(T_{i \lambda}^{\bar{j}}+T_{\lambda j}^{\bar{i}}-T_{j i}^{\bar{\lambda}}\right)+\frac{1}{2} T_{\bar{k} \bar{l}}^{\bar{\lambda}} T_{i j}^{\lambda} \\
= & R_{\bar{k} \overline{l i j}}-\frac{1}{2} \nabla_{\bar{k}} T_{j i}^{l}+\frac{1}{2} \nabla_{\bar{l}} T_{j i}^{k}+\frac{1}{4} T_{i \lambda}^{l} T_{\bar{\lambda} \bar{k}}^{\bar{j}}-\frac{1}{4} T_{\bar{l} \bar{\lambda}}^{\bar{i}} T_{j \lambda}^{k}-\frac{1}{4} T_{i \lambda}^{k} T_{\bar{\lambda} \bar{l}}^{\bar{j}}+\frac{1}{4} T_{\bar{k} \bar{\lambda}}^{\bar{i}} T_{j \lambda}^{l} \\
& +\frac{1}{2} T_{\bar{k} \bar{l}}^{\lambda}\left(T_{i \lambda}^{\bar{j}}+T_{\lambda j}^{\bar{i}}-T_{j i}^{\bar{\lambda}}\right)+\frac{1}{2} T_{\bar{k} \bar{l}}^{\bar{\lambda}} T_{i j}^{\lambda} \\
= & -\frac{1}{2} \nabla_{\bar{k}} T_{j i}^{l}+\frac{1}{2} \nabla_{\bar{l}} T_{j i}^{k}+\frac{1}{4} T_{i \lambda}^{l} T_{\bar{\lambda} \bar{k}}^{\bar{j}}-\frac{1}{4} T_{\bar{l} \bar{\lambda}}^{\bar{i}} T_{j \lambda}^{k}-\frac{1}{4} T_{i \lambda}^{k} T_{\bar{\lambda} \bar{l}}^{\bar{j}}+\frac{1}{4} T_{\bar{k} \bar{\lambda}}^{\bar{i}} T_{j \lambda}^{l} \\
& +\frac{1}{2} T_{\bar{k} \bar{l}}^{\lambda}\left(T_{i \lambda}^{\bar{j}}+T_{\lambda j}^{\bar{i}}-T_{j i}^{\bar{\lambda}}\right)+\frac{1}{2} T_{\bar{k} \bar{l}}^{\bar{\lambda}} T_{i j}^{\lambda},
\end{aligned}
$$

where we used the definitions (4.9), (4.10), the formula (4.1), and that $\nabla_{\left[Z_{\bar{k}}, Z_{\bar{l}}\right]} Z_{i}(p)=0$, and $R_{\bar{k} \overline{l i j}}=0$.

By taking $k=i, l=j$ and sum them over, we obtain at $p$,

$$
\begin{aligned}
R_{i j \bar{i} j}^{L}= & -\frac{1}{2} \nabla_{\bar{i}} T_{j i}^{j}+\frac{1}{2} \nabla_{\bar{j}} T_{j i}^{i}+\frac{1}{4} T_{i \lambda}^{j} T_{\bar{\lambda} \bar{i}}^{\bar{j}}-\frac{1}{4} T_{\bar{j} \bar{\lambda}}^{\bar{i}} T_{j \lambda}^{i}-\frac{1}{4} T_{i \lambda}^{i} T_{\bar{\lambda} \bar{j}}^{\bar{j}}+\frac{1}{4} T_{\bar{i} \bar{\lambda}}^{\bar{i}} T_{j \lambda}^{j} \\
& +\frac{1}{2} T_{\bar{i} j}^{\lambda}\left(T_{i \lambda}^{\bar{j}}+T_{\lambda j}^{\bar{i}}-T_{j i}^{\bar{\lambda}}\right)+\frac{1}{2} T_{\bar{i} \bar{j}}^{\bar{\lambda}} T_{i j}^{\lambda} \\
= & \frac{1}{2} \nabla_{\bar{i}} w_{i}+\frac{1}{2} \nabla_{\bar{j}} w_{j}-\frac{1}{4} T_{i \lambda}^{j} T_{\bar{i} \bar{\lambda}}^{\bar{j}}-\frac{1}{4} T_{\bar{j} \bar{\lambda}}^{\bar{i}} T_{j \lambda}^{i}+\frac{1}{4} T_{\lambda i}^{i} T_{\bar{\lambda} \bar{j}}^{\bar{j}}+\frac{1}{4} T_{\bar{\lambda} \bar{i}}^{\bar{i}} T_{\lambda j}^{j} \\
& +\frac{1}{2} T_{\bar{i} j}^{\lambda} T_{i \lambda}^{\bar{j}}+\frac{1}{2} T_{\overline{j i}}^{\lambda} T_{j \lambda}^{\bar{i}}+\frac{1}{2} T_{\bar{i} \bar{j}}^{\lambda} T_{i j}^{\bar{\lambda}}+\frac{1}{2} T_{\bar{i} \bar{j}}^{\bar{\lambda}} T_{i j}^{\lambda} \\
= & \nabla_{\bar{i}} w_{i}-\frac{1}{2}\left|T^{\prime}\right|^{2}+\frac{1}{2}|w|^{2}+T_{\bar{i} \bar{j}}^{\lambda} T_{\lambda j}^{\bar{i}}+\frac{1}{2}\left|T^{\prime \prime}\right|^{2}+\frac{1}{2}\left|T^{\prime}\right|^{2} \\
= & \nabla_{\bar{i}} w_{i}+\frac{1}{2}|w|^{2}+B_{\bar{i} \bar{j}}^{\lambda} B_{\lambda j}^{\bar{i}}+\frac{1}{2}\left|T^{\prime \prime}\right|^{2},
\end{aligned}
$$


where we used that $T_{i j}^{\bar{k}}=-B_{i j}^{\bar{k}}$. Since we have assumed that $g$ is almost balanced, which is equivalent to that $B_{i k}^{\bar{q}} B_{\bar{q} \bar{l}}^{i}=0$ for all $k, l=1, \ldots, n$ on a compact Kähler-like almost Hermitian manifold from Proposition 1.2, then especially we have that $B_{i j}^{\lambda} B_{\lambda j}^{\bar{i}}=0$. Also we have $w_{r}=0$ for all $r=1, \ldots, n$ since it is equivalent to the almost balancedness from Lemma 4.3. Combining these, we get $R_{i j i j}^{L}=\frac{1}{2}\left|T^{\prime \prime}\right|^{2}$. In addition, since we have assumed that $M$ is G-Kähler-like as well, we then obtain at $p$,

$$
0=R_{i j \overline{i j}}^{L}=\frac{1}{2}\left|T^{\prime \prime}\right|^{2},
$$

which implies that we have that $T_{i j}^{\bar{k}}(p)=0$ for all $i, j$ and $k$. Since the point $p$ was chosen arbitrary, we obtain that $T_{i j}^{\bar{k}}=0$ for all $i, j$ and $k$ on whole $M$, which is equivalent to that $J$ is integrable. Then we may apply Proposition 1.4, and we conclude that $g$ is Kähler.

Next, we give a proof of Theorem 1.2.

Proof of Theorem 1.2. As in the proof of Theorem 1.1, for an arbitrary chosen fixed point $p \in M$, we choose a local $(1,0)$-frame $\left\{Z_{r}\right\}$ at $p$ such that $\nabla Z_{r}(p)=0$ and $g_{i j}(p)=\delta_{i j}$. As in the proof of Theorem 1.1, we use the formula (4.23), and since we have assumed the G-Kähler-likeness, we have at $p$,

$$
0=R_{i j \bar{i} \bar{j}}^{L}=\nabla_{\bar{i}} w_{i}+B_{\overline{i j}}^{\lambda} B_{\lambda j}^{\bar{i}}+\frac{1}{2}|w|^{2}+\frac{1}{2}\left|T^{\prime \prime}\right|^{2} .
$$

Since the fixed point $p$ was chosen arbitrary, the equation

$$
0 \leq|w|^{2}+\left|T^{\prime \prime}\right|^{2}=-2\left(\nabla_{\bar{i}} w_{i}+B_{i j}^{\lambda} B_{\lambda j}^{\bar{i}}\right) \leq 0
$$

holds on whole $M$, since we have assumed that $\nabla_{\bar{i}} w_{i}+B_{i j}^{\lambda} B_{\lambda j}^{\bar{i}} \geq 0$. Then we get that $w=0$ and $T^{\prime \prime}=0$, which tell us the desired result since $g$ is almost balanced if and only if $w_{i}=0$ for all $i=1, \ldots, n$, and also since $J$ is integrable if and only if $T^{\prime \prime}$ vanishes.

Now, we prove Corollary 3.3.

Proof of Corollary 3.3. For an arbitrary chosen fixed point $p \in M$, we choose a local $(1,0)$-frame $\left\{Z_{r}\right\}$ at $p$ such that $\nabla Z_{r}(p)=0$ and $g_{i j}(p)=\delta_{i j}$. From Lemma 4.4, we have that the torsion $(1,0)$-form $\eta$ satisfies $\operatorname{Re}(\bar{\partial} \eta)=0$ is equivalent to that the metric $g$ is almost Gauduchon. Note that $\operatorname{Re}(\bar{\partial} \eta)=0$ implies that $\operatorname{Re}\left(\nabla_{\bar{l}} w_{k}\right)=0$ for all $k, l=1, \ldots, n$, especially we then have $\operatorname{Re}\left(\nabla_{\bar{i}} w_{i}\right)=0$. As in the proof of Theorem 1.1 and 1.2, we again use the formula (4.23), and since we have assumed the G-Kähler-likeness, we have at $p$,

$$
\begin{aligned}
& 0=R_{i j i \bar{j}}^{L}=\nabla_{\bar{i}} w_{i}+B_{i j}^{\lambda} B_{\lambda j}^{\bar{i}}+\frac{1}{2}|w|^{2}+\frac{1}{2}\left|T^{\prime \prime}\right|^{2} \\
\Leftrightarrow \quad & \nabla_{\bar{i}} w_{i}=-\left(B_{i j}^{\lambda} B_{\lambda j}^{\bar{i}}+\frac{1}{2}|w|^{2}+\frac{1}{2}\left|T^{\prime \prime}\right|^{2}\right),
\end{aligned}
$$

which implies that $\nabla_{\bar{i}} w_{i}=\overline{\nabla_{\bar{i}} w_{i}}$, that is, $\nabla_{\bar{i}} w_{i}=\operatorname{Re}\left(\nabla_{\bar{i}} w_{i}\right)=0$ since we have that

$$
\overline{B_{\bar{i} j}^{\lambda} B_{\lambda j}^{\bar{i}}}=B_{i j}^{\bar{\lambda}} B_{\bar{\lambda} \bar{j}}^{i}=B_{i \bar{j}}^{\lambda} B_{\lambda j}^{\bar{i}} \text {. }
$$

Hence we obtain that

$$
B_{i j}^{\lambda} B_{\lambda j}^{\bar{i}}+\frac{1}{2}|w|^{2}+\frac{1}{2}\left|T^{\prime \prime}\right|^{2}=0 .
$$

Since the fixed point $p$ was chosen arbitrary, the equation

$$
0 \leq|w|^{2}+\left|T^{\prime \prime}\right|^{2}=-2 B_{i \bar{j}}^{\lambda} B_{\lambda j}^{\bar{i}} \leq 0
$$

holds on whole $M$, since we have assumed that $B_{i j}^{\lambda} B_{\lambda j}^{\bar{i}} \geq 0$. Then we get that $w=0$ and $T^{\prime \prime}=0$, which tell us the desired result.

Next, we prove Corollary 3.4 . 
Proof of Corollary 3.4. Let $(M, J, g)$ be a G-Kähler-like quasi-Kähler manifold satisfying $B_{i j}^{\lambda} B_{\lambda j}^{\bar{i}} \geq 0$. For an arbitrary chosen fixed point $p \in M$, we choose a local $(1,0)$-frame $\left\{Z_{r}\right\}$ at $p$ such that $\nabla Z_{r}(p)=0$ and $g_{i j}(p)=\delta_{i j}$. From Lemma 3.4, we have that $T_{i j}^{k}=0$ for all $i, j, k=1, \ldots, n$, especially we then have $w_{i}=0$ for all $i=1, \ldots, n$. As we see in the proof of Theorem 1.1 and 1.2, since we have assumed the G-Kähler-likeness, we have at $p$,

$$
0=R_{i j i \bar{j}}^{L}=B_{i j}^{\lambda} B_{\lambda j}^{\bar{i}}+\frac{1}{2}\left|T^{\prime \prime}\right|^{2} .
$$

Since the fixed point $p$ was chosen arbitrary, the equation

$$
0 \leq\left|T^{\prime \prime}\right|^{2}=-2 B_{\overline{i j}}^{\lambda} B_{\lambda j}^{\bar{i}} \leq 0
$$

holds on whole $M$, since we have assumed that $B_{\bar{i}}^{\lambda} B_{\lambda j}^{\bar{i}} \geq 0$. Then we get that $T^{\prime \prime}=0$, which tells us that $J$ is integrable. Therefore, $g$ is Kähler.

\section{Proof of Theorem 3.1}

Let $\left(M^{2 n}, J, g\right)$ be a compact almost Hermitian manifold. Let $\nabla$ be the Chern connection withrespect to $g$ on $\left(M^{2 n}, J, g\right)$. Let $\left\{Z_{r}\right\}$ be a local unitary $(1,0)$-frame with respect to $g$ around a fixed point $p \in M$. Note that unitary frames always exist locally since we can take any frame and apply the Gram-Schmidt process. Then with respect to a local $g$-unitary frame, we have $g_{i j}=\delta_{i j}, Z_{k}\left(g_{i j}\right)=0$ for any $i, j, k=1, \ldots, n$, and the Christoffel symbols satisfy $\Gamma_{i j}^{k}=-\Gamma_{i \bar{k}}^{\bar{j}}=-B_{i \bar{k}}^{\bar{j}}, \Gamma_{\overline{j k}}^{S}=\overline{\Gamma_{j \bar{k}}^{\bar{S}}}=-\overline{\Gamma_{j s}^{k}}=-\Gamma_{\bar{j} \bar{s}}^{\bar{k}}$. Then we have

$$
\begin{aligned}
& \overline{R_{i \bar{j} k}^{r}}=Z_{\bar{i}}\left(\Gamma_{j \bar{k}}^{\bar{r}}\right)-Z_{j}\left(\Gamma_{\bar{i} \bar{k}}^{\bar{r}}\right)+\Gamma_{\bar{i} \bar{s}}^{\bar{r}} \Gamma_{j \bar{k}}^{\bar{s}}-\Gamma_{j \bar{s}}^{\bar{r}} \Gamma_{\bar{i} \bar{k}}^{\bar{s}}-B_{\bar{i}}^{\bar{s}} \Gamma_{\bar{s} \bar{k}}^{\bar{r}}+B_{j i}^{s} \Gamma_{s \bar{k}}^{\bar{r}} \\
& =Z_{j}\left(\Gamma_{\bar{i} r}^{k}\right)-Z_{\bar{i}}\left(\Gamma_{j r}^{k}\right)+\Gamma_{\bar{i} r}^{s} \Gamma_{j s}^{k}-\Gamma_{j r}^{s} \Gamma_{\bar{i} s}^{k}-B_{j i}^{s} \Gamma_{s r}^{k}+B_{i j}^{\bar{s}} \Gamma_{\bar{s} r}^{k} \\
& =R_{j \overline{i r}}{ }^{k} \text {, }
\end{aligned}
$$

hence we obtain $\overline{R_{i \bar{j} k \bar{r}}}=R_{j \bar{i} \bar{k} \bar{k}}$ by using a local unitary frame with respect to $g$.

In order to prove Theorem 3.1, we prepare the following lemma.

Lemma 5.1. ([24, Theorem 3.1]) Let $(M, J, g)$ be an almost Hermitian manifold and fix a local unitary $(1,0)$ frame. Then,

$$
\begin{aligned}
R_{i j k \bar{l}}^{L}= & \frac{1}{2}\left(R_{i \bar{l} k \bar{j}}+R_{k \overline{j i} \bar{l}}\right)-\frac{1}{4}\left(T_{\bar{l} \bar{\lambda}}^{\bar{i}} T_{k \lambda}^{j}+T_{i \lambda}^{l} T_{\bar{j} \bar{\lambda}}^{\bar{k}}-T_{i k}^{\lambda} T_{\bar{j} \bar{l}}^{\bar{\lambda}}\right) \\
& -\frac{1}{2}\left(T_{k \lambda}^{\bar{i}} T_{\bar{j} \bar{l}}^{\lambda}+T_{i k}^{\bar{\lambda}} T_{\bar{l} \bar{\lambda}}^{j}\right)+\frac{1}{4}\left(T_{i k}^{\bar{\lambda}}+T_{k \lambda}^{\bar{i}}-T_{\lambda i}^{\bar{k}}\right)\left(T_{\bar{j} \bar{l}}^{\lambda}+T_{\bar{l} \bar{\lambda}}^{j}-T_{\bar{\lambda} \bar{j}}^{l}\right) .
\end{aligned}
$$

Proof of Theorem 3.1. Let $(M, J, g)$ be a G-Kähler-like almost Hermitian manifold with $n \geq 2$. We assume that $R_{i \bar{j} k \bar{l}}=R_{i \bar{j} k \bar{l}}^{L}$ for all $i, j, k, l=1, \ldots, n$ and $B_{\bar{i} j}^{\lambda} B_{\lambda j}^{\bar{i}} \geq 0$. Let $\left\{Z_{r}\right\}$ be a local unitary $(1,0)$-frame with respect to $g$. From Lemma 3.2, we have that $(M, J, g)$ is Kähler-like. By taking $j=i, l=k$ and sum them over, we have

$$
\begin{aligned}
R_{i \bar{i} k \bar{k}}^{L}= & R_{i \bar{i} k \bar{k}}-\frac{1}{4}\left(T_{k \lambda}^{i} T_{\bar{k} \bar{\lambda}}^{\bar{i}}+T_{i \lambda}^{k} T_{\bar{i} \overline{\bar{\lambda}}}^{\bar{k}}-T_{i k}^{\lambda} T_{\bar{i} \bar{k}}^{\bar{\lambda}}\right)-\frac{1}{2}\left(T_{k \lambda}^{\bar{i}} T_{\bar{i} \bar{k}}^{\lambda}+T_{i k}^{\bar{\lambda}} T_{\bar{k} \bar{\lambda}}^{i}\right) \\
& +\frac{1}{4}\left(T_{i k}^{\bar{\lambda}}+T_{k \lambda}^{\bar{i}}-T_{\lambda i}^{\bar{k}}\right)\left(T_{\bar{i} \bar{k}}^{\lambda}+T_{\bar{k} \bar{\lambda}}^{i}-T_{\bar{\lambda} \bar{i}}^{k}\right),
\end{aligned}
$$

where we used that $R_{k \bar{i} \bar{k}}=R_{\bar{i} \bar{k} \bar{k}}$. Since we have assumed that $R_{i \bar{j} k \bar{l}}=R_{i \bar{j} k \bar{l}}^{L}$ for all $i, j, k, l=1, \ldots, n$, we obtain

$$
0=-\frac{1}{4}\left|T^{\prime}\right|^{2}-\frac{1}{2}\left(B_{k \lambda}^{\bar{i}} B_{\bar{i} \bar{k}}^{\lambda}+B_{i k}^{\bar{\lambda}} B_{\bar{k} \bar{\lambda}}^{i}\right)+\frac{1}{4}\left|B_{i k}^{\bar{\lambda}}+B_{k \lambda}^{\bar{i}}-B_{\lambda i}^{\bar{k}}\right|^{2},
$$

where we used that $T_{i k}^{\bar{\lambda}}=-B_{i k}^{\bar{\lambda}}$. Note that we have $B_{k \lambda}^{\bar{i}}=-B_{\lambda k}^{\bar{i}}$ and then we get $B_{k \lambda}^{\bar{i}} B_{\bar{i} \bar{k}}^{\lambda}+B_{i k}^{\bar{\lambda}} B_{\bar{k} \bar{\lambda}}^{i}=-2 B_{\lambda k}^{\bar{i}} B_{\bar{i} \bar{k}}^{\lambda}$. As a result, we can rewrite (5.4) as

$$
\left|T^{\prime}\right|^{2}=4 B_{\lambda k}^{\bar{i}} B_{\bar{i} \bar{k}}^{\lambda}+\left|B_{i k}^{\bar{\lambda}}+B_{k \lambda}^{\bar{i}}-B_{\lambda i}^{\bar{k}}\right|^{2} .
$$


Actually, the right-hand side of (5.5) is equal to zero;

$$
4 B_{\lambda k}^{\bar{i}} B_{\bar{i} \bar{k}}^{\lambda}+\left|B_{i k}^{\bar{\lambda}}+B_{k \lambda}^{\bar{i}}-B_{\lambda i}^{\bar{k}}\right|^{2}=0,
$$

since we have $B_{i k}^{\bar{\lambda}}=-T_{i k}^{\bar{\lambda}}=0$ from Corollary 1.2. Recall that Corollary 1.2 states that if $(M, J, g)$ is a Kählerlike and G-Kähler-like almost Hermitian manifold with $B_{\overline{i j}}^{\lambda} B_{\lambda j}^{\bar{i}} \geq 0$, then $J$ is integrable. Here notice that $J$ is integrable if and only if $T^{\prime \prime}$ vanishes. Therefore we conclude that, by applying Corollary 1.2, under the assumptions in Theorem 3.1, we have that $J$ is integrable and $\left|T^{\prime}\right|^{2}=0$ at the same time. Since $T^{\prime}=0$ is equivalent to that $(M, J, g)$ is quasi-Kähler (Lemma 3.4), and since the quasi-Kählerity indicates the Kählerity when $J$ is integrable, we obtain the desired result.

\section{References}

[1] Abbena E., Garbiero S., Salamon S. Almost Hermitian geometry on six dimensional nilmanifolds Ann. Scuola Norm. Sup. Pisa Cl. Sci. (4) 30 (2001), no. 1, 147-170.

[2] Agricola, I., Borówka, A., Friedrich, T. $S^{6}$ and the geometry of nearly Kähler 6-manifolds, Diff. Geom. and its Appl. 57 (2018) 75-86.

[3] Angella, D., Otal, A., Ugarte, L., Villacampa, R. On Gauduchon connections with Kähler-like curvature, preprint, arXiv:1809.02632v1.

[4] Chern, S.-S. Characteristic classes of Hermitian manifolds, Ann. of Math. 47 (1946) 85-121.

[5] Di Scala, A. J., Vezzoni, L. Gray identities, canonical connection and integrability, Proc. of the Edinburgh Math. Soc. 53 (2010) 657-674.

[6] Di Scala, A. J., Vezzoni, L. Chern-flat and Ricci-flat invariant almost Hermitian structures, Ann. Glob. Anal. Geom. 40 (2011) 21-45.

[7] Di Scala, A. J., Vezzoni, L. Quasi-Kähler manifolds with trivial Chern holonomy, Math. Z. 271 (2012) 95-108.

[8] Gauduchon, P. Le thèorème de l'excentricité nulle, C. R. Acad. Sci. Paris Sér. A-B 285 (1977), no. 5, A387-A390.

[9] Gauduchon, P. Hermitian connections and Dirac operators, Boll. Un. Mat. Ital. B (7) (suppl.) 11 (2) (1997) 257-288.

[10] Gray, A. Some examples of almost Hermitian manifolds, Illinois J. Math. 10 (1966), 353-366.

[11] Gray, A. Nearly Kähler manifolds, J. Diff. Geom. 4 (1970), 283-309.

[12] Gray, A. Curvature identities for Hermitian and almost Hermitian manifolds, Tohoku Math. J. (2) 28 (1976), no. 4, $601-612$.

[13] Hsiung, C C. Almost complex and complex structures, World Scientific, Series in Pure Mathematics-Volume 20, (1995), ISBN 981-02-1712-9.

[14] Kawamura, M. An almost pluriclosed flow, Geometric Flows 2 (2017), no. 1, 72-85.

[15] Kawamura, M. On Kähler-like almost Hermitian metrics and the almost Hermitian curvature flow, to appear in Hokkaido Math. J.

[16] Kawamura, M. On the Kähler-likeness on almost Hermitian manifolds, Complex Manifolds 6 (2019), 366-376.

[17] Liu, K., Yang, X. Ricci curvatures on Hermitian manifolds, Trans. Amer. Math. Soc. 369 (2017), 5157-5196.

[18] Newlander, A., Nirenberg, L. Complex analytic coordinates in almost complex manifolds, Ann. of Math. (2) 65 (1957), $391-404$.

[19] Popovici, D. Aeppli cohomology classes associated with Gauduchon metrics on compact complex manifolds, Bulletin de la Société Mathématique de France, 143 (2015), no. 4, 763-800.

[20] Tosatti, V., Weinkove, B., Yau, S-T. Taming symplectic forms and the Calabi-Yau equation, Proc. Lond. Math. Soc. 97 (2008), no. 2, 401-424.

[21] Vaisman, I. Some curvature properties of complex surfaces, Ann. Mat. Pura Appl. (4) 132 (1982), 1-18 (1983).

[22] Vezzoni, L. On Hermitian curvature flow on almost complex manifolds, Diff. Geom. and its Appl. 29 (2011), 709-722.

[23] Yang, B. and Zheng, F. On curvature tensors of Hermitian manifolds, Comm. Anal. Geom. (5) 26 (2018), 1195-1222.

[24] Yu C.-J. Curvature identities on almost Hermitian manifolds and applications, Sci. China Math. 60 (2017), Issue 2: $285-300$.

[25] Yu C.-J. Nonpositively curved almost Hermitian metrics on products of compact almost complex manifolds, Acta Mathematica Sinica, 31 (2015), no. 1, 61-70.

[26] Zheng, T. An almost complex Chern-Ricci flow, J. Geom. Anal. (2017). https://doi.org/10.1007/s12220-017-9898-9. 